\title{
Design of Artificial Neural Networks for the Novel Applications of the Sixth Order Singular Nonlinear Pantograph Engineering Model
}

\section{Zulqurnain Sabir}

Hazara University

Mohamed Ali ( $\square$ mohamed.reda@bhit.bu.edu.eg )

Benha University

Muhammad Asif Zahoor Raja

National Yunlin Institute of Technology: National Yunlin University of Science and Technology

R. Sadat

Zagazig University

Original Article

Keywords: Sixth order model, Pantograph, Singular, Shape factors, Levenberg-Marquardt backpropagation

Posted Date: July 20th, 2021

DOI: https://doi.org/10.21203/rs.3.rs-718926/v1

License: (c) (i) This work is licensed under a Creative Commons Attribution 4.0 International License. Read Full License 


\title{
Design of artificial neural networks for the novel applications of the sixth order singular nonlinear pantograph engineering model
}

\author{
Zulqurnain Sabir ${ }^{1}$, Mohamed R. Ali ${ }^{*}$, Muhammad Asif Zahoor Raja ${ }^{3}$, R. Sadat ${ }^{4}$ \\ ${ }^{1}$ Department of Mathematics and Statistics, Hazara University, Mansehra, Pakistan \\ Email: zulqarnain_maths@hu.edu.pk \\ ${ }^{2}$ Basic Engineering Mathematics Department. \\ Benha Faculty of Engineering, Benha University- Egypt. \\ Email: mohamed.reda@bhit.bu.edu.eg \\ ${ }^{3}$ Future Technology Research Center, National Yunlin University of Science and Technology, 123 University Road, \\ Section 3, Douliou, Yunlin 64002, Taiwan, R.O.C. \\ Email: rajemaz@yuntech.edu.tw \\ ${ }^{4}$ Department of Mathematics, Zagazig Faculty of Engineering, Zagazig University, Egypt
}

Corresponding author: Mohamed R. Ali

Email: mohamed.reda@bhit.bu.edu.eg

\begin{abstract}
This aim of this study is to design and numerically investigate a novel nonlinear singular sixth order (SSO) pantograph differential system (PDS), i.e., SSO-PDS along with its five categories. The design of the novel nonlinear SSO-PDS is presented using the concepts of the standard Emden-Fowler along with the pantograph differential system. The singular systems are applied in many engineering and mathematical applications, like inverse models and viscoelasticity systems. The shape factor, singular point and pantograph terms are provided for each category of the novel nonlinear SSO-PDS. The numerical investigations of the nonlinear SSO-PDS are presented through the artificial neural networks (ANNs) using the LevenbergMarquardt backpropagation (LMB), i.e., ANNs-LMB. The proposed results have been compared with the reference solutions to check the correctness of the designed novel model. The proposed solutions of the novel SSO-PDS are assessed using the training, testing and verification procedures in the form of mean square error (MSE). For the capability, efficacy and accuracy of the novel SSO-PDS through the ANNs, the numerical results are drawn using the MSE, error histograms and regression as well as correlation graphs.
\end{abstract}

Keywords: Sixth order model; Pantograph; Singular; Shape factors; Levenberg-Marquardt backpropagation

\section{Introduction}

The singular systems have various applications in the mathematical and engineering areas. The ordinary differential systems have much significance due to the wide range of applications in scientific and engineering fields, e.g., chemical reactor systems, biological applications, astrophysics, nonlinear circuits, control-based optimization theory, theory of boundary layer and fluidics [1-6]. The present investigations are associated to the Emden-Fowler system (EFS) that is famous due to singularity. The EFS are considered complex and difficult to solve because of the 
stiffer nature. It is not easy to solve the EFS using the numerical and analytical schemes. The EFS has a variety of applications in the fields of engineering, relativistic mechanics, population evolution system, pattern creation networks and chemical reactor [7-10]. The mathematical form of the EFS is provided as [11-13]:

$$
\begin{aligned}
& \frac{d^{2} \omega}{d q^{2}}+\frac{\delta}{q} \frac{d \omega}{d q}+f(q) h(\omega)=k(q), \\
& \omega(0)=a, \frac{d \omega}{d q}(0)=0
\end{aligned}
$$

where, $\delta \geq 1$ is the shape factor, $k(q)$ is the forcing factor. The EFS becomes the Lane-Emden system (LES) by taking $k(q)=1$, given as:

$$
\begin{aligned}
& \frac{d^{2} \omega}{d q^{2}}+\frac{\delta}{q} \frac{d \omega}{d q}+h(\omega)=k(q) \\
& \omega(0)=a, \frac{d \omega}{d q}(0)=0
\end{aligned}
$$

The LES has great history and has many applications in the gas cloud model, radiative cooling operators, stellar process, mathematical physics systems, cluster galaxies and structures of polytropic stars [14-15]. The term $h(\omega)=\omega^{z}$ shows different forms for different values of $z$. For $z$ $=0$ and 1 , the LES represents the linear form, otherwise it is nonlinear. For $h(\omega)=e^{\omega}$, the LES represents the form of isothermal gas sphere. Furthermore, $h(\omega)$ represents the nonlinearity as $\cosh u, \cos u, \sinh u$ and $\sin u$, etc. The mathematical form of the Eq (2) becomes white-dwarf system for $h(\omega)=\left(\omega^{2}-C\right)^{1.5}$ introduced by Chandrasekhar [16]. Few well known applications of LES are the mathematical physics [17], electromagnetics [18], density profile of gaseous star [19], oscillating magnetic areas [20], quantum mechanical networks [21], isotropic state of continuous media [22], sublinear neutral span [23] and system of stellar structure [24]. A number of techniques have been proposed to solve such singular systems since their invention [25-33].

The differential model based on the pantograph has huge implication due to its huge importance in the areas of engineering, science and biology. Few mention applications are communication models, light absorption in the stellar solid, dynamical population systems, control systems, infectious diseases, propagation models, electronic networks, transportations and quantum mechanics [34-39]. A variety of schemes have been implemented to solve the pantograph types of system like the one-dimensional transformation method, Direchlet series approach, the Taylor polynomial scheme [40-45]. In order to solve the singular systems, few common schemes have been presented like the Haar Adomian method is presented by Saeed et al [47], the series scheme is implemented by Romas et al [46], the differential transformation scheme is explored by Sabir et al [49] and the reproduced kernel approach applied by Hashemi et al [48]. Moreover, the singular system has been numerically treated by the swarming/heuristic approaches have been given in these citations [50-53]. 
The aim of this study is to present the numerical performances of the novel nonlinear singular sixth order pantograph differential system (SSO-PDS) along with its five categories based on the artificial neural networks (ANNs) using the Levenberg-Marquardt backpropagation (LMB), i.e., ANNs-LMB. The numerical simulations of each case are presented and discuss in detail to show the correctness of the designed model. Few novel features of this study are presented as:

- A nonlinear novel SSO-PDS along with its five categories is presented using the celebrated Emden-Fowler and pantograph differential system.

- The detail of the singular points (SPs), shape factors (SFs) and the pantograph factors (PFs) is provided to solve each category of the nonlinear novel SSO-PDS.

- The numerical results of the nonlinear novel SSO-PDS have been presented using the stochastic procedures based on the ANNs-LMB.

- The comparison of the obtained results through the ANNs-LMB are performed in good measures with the reference solutions.

- The matching of the obtained and reference numerical performances indicates the worth of the stochastic ANNs-LMB to solve each category of the nonlinear novel SSO-PDS.

- The stochastic ANNs-LMB routine via relative soundings based on the error histograms (EHs), regression/correlation metrics and mean square error (MSE) indicates the worth of the designed approach.

This research work is detailed as follows: The structure of the nonlinear novel SSO-PDS with five classes is provided in Sect 2. The designed methodology is provided in Sect 3. The detail of the results through ANNs-LMB is provided in Sect 4. The conclusion and reports based on future research are provided in the final Sect.

\section{Construction of the nonlinear novel SSO-PDS}

In this section, the structure of the nonlinear novel SSO-PDS along with its five types and their initial conditions (ICs) is provided by using the traditional form of the EFS. To derive the nonlinear novel SSO-PDS, the mathematical form is given as:

$$
\left\{\begin{array}{l}
q^{-\Phi_{1}} \frac{d^{x}}{d q^{x}}\left(q^{\Phi_{1}} \frac{d^{w}}{d q^{w}}\right) \omega_{1}(\chi q)+h_{1}(q) f_{1}\left(\omega_{1}, \omega_{2}\right)=k_{1}(q), \\
q^{-\Phi_{2}} \frac{d^{x}}{d q^{x}}\left(q^{\Phi_{2}} \frac{d^{w}}{d q^{w}}\right) \omega_{2}(\chi q)+h_{2}(q) f_{2}\left(\omega_{1}, \omega_{2}\right)=k_{2}(q),
\end{array}\right.
$$

where $\Phi_{1}$ and $\Phi_{2}$ are the positive terms, $h_{1}(q)$ and $h_{2}(q)$ indicate the values of the functions, $f_{1}\left(\omega_{1}, \omega_{2}\right)$ and $f_{2}\left(\omega_{1}, \omega_{2}\right)$ represent the linear/nonlinear functions of $\omega_{1}$ and $\omega_{2}$, the forcing functions are $k_{1}(q)$ and $k_{2}(q)$. The values of the $x$ and $w$ shown in Eq 3 are designated as:

$$
x+w=6, \quad x, w \geq 1 .
$$

For the above equation, the following five possibilities are provided as: 


$$
\begin{aligned}
& x=5, w=1, \\
& x=4, w=2, \\
& x=3, w=3, \\
& x=2, w=4, \\
& x=1, w=5,
\end{aligned}
$$

Type 1: The updated mathematical form of Eq (3) with $x=5$ and $w=1$ is given as:

$$
\left\{\begin{array}{l}
q^{-\Phi_{1}} \frac{d^{5}}{d q^{5}}\left(q^{\Phi_{1}} \frac{d}{d q}\right) \omega_{1}(\chi q)+h_{1}(q) f_{1}\left(\omega_{1}, \omega_{2}\right)=k_{1}(q), \\
q^{-\Phi_{2}} \frac{d^{5}}{d q^{5}}\left(q^{\Phi_{2}} \frac{d}{d q}\right) \omega_{2}(\chi q)+h_{2}(q) f_{2}\left(\omega_{1}, \omega_{2}\right)=k_{2}(q),
\end{array}\right.
$$

Solving the above derivative as:

$$
\left\{\begin{array}{l}
\frac{d^{5}}{d q^{5}}\left(q^{\Phi_{1}} \frac{d}{d q}\right) \omega_{1}(\chi q)=\chi^{5} q^{\Phi_{1}} \frac{d^{6} \omega_{1}(\chi q)}{d q^{6}}+5 \chi^{4} \Phi_{1} q^{\Phi_{1}-1} \frac{d^{5} \omega_{1}(\chi q)}{d q^{5}}+10 \chi^{3} \Phi_{1}\left(\Phi_{1}-1\right) q^{\Phi_{1}-2} \frac{d^{4} \omega_{1}(\chi q)}{d q^{4}} \\
+10 \chi^{2} \Phi_{1}\left(\Phi_{1}-1\right)\left(\Phi_{1}-2\right) q^{\Phi_{1}-3} \frac{d^{3} \omega_{1}(\chi q)}{d q^{3}}+5 \chi \Phi_{1}\left(\Phi_{1}-1\right)\left(\Phi_{1}-2\right)\left(\Phi_{1}-3\right) q^{\Phi_{1}-4} \frac{d^{2} \omega_{1}(\chi q)}{d q^{2}} \\
+\Phi_{1}\left(\Phi_{1}-1\right)\left(\Phi_{1}-2\right)\left(\Phi_{1}-3\right)\left(\Phi_{1}-4\right) q^{\Phi_{1}-5} \frac{d \omega_{1}(\chi q)}{d q}, \\
\frac{d^{5}}{d q^{5}}\left(q^{\Phi_{2}} \frac{d}{d q}\right) \omega_{2}(\chi q)=\chi^{5} q^{\Phi_{2}} \frac{d^{6} \omega_{2}(\chi q)}{d q^{6}}+5 \chi^{4} \Phi_{2} q^{\Phi_{2}-1} \frac{d^{5} \omega_{2}(\chi q)}{d q^{5}}+10 \chi^{3} \Phi_{2}\left(\Phi_{2}-1\right) q^{\Phi_{2}-2} \frac{d^{4} \omega_{2}(\chi q)}{d q^{4}} \\
+10 \chi^{2} \Phi_{2}\left(\Phi_{2}-1\right)\left(\Phi_{2}-2\right) q^{\Phi_{2}-3} \frac{d^{3} \omega_{2}(\chi q)}{d q^{3}}+5 \chi \Phi_{2}\left(\Phi_{2}-1\right)\left(\Phi_{2}-2\right)\left(\Phi_{2}-3\right) q^{\Phi_{2}-4} \frac{d^{2} \omega_{2}(\chi q)}{d q^{2}} \\
+\Phi_{2}\left(\Phi_{2}-1\right)\left(\Phi_{2}-2\right)\left(\Phi_{2}-3\right)\left(\Phi_{2}-4\right) q^{\Phi_{2}-5} \frac{d \omega_{2}(\chi q)}{d q} .
\end{array}\right.
$$

By using Eqs (10) and (11), the updated form of the nonlinear novel SSO-PDS is given as: 


$$
\left\{\begin{array}{l}
\chi^{5} \frac{d^{6} \omega_{1}(\chi q)}{d q^{6}}+\frac{5 \chi^{4} \Phi_{1}}{q} \frac{d^{5} \omega_{1}(\chi q)}{d q^{5}}+\frac{10 \chi^{3} \Phi_{1}\left(\Phi_{1}-1\right)}{q^{2}} \frac{d^{4} \omega_{1}(\chi q)}{d q^{4}}+\frac{10 \chi^{2} \Phi_{1}\left(\Phi_{1}-1\right)\left(\Phi_{1}-2\right)}{q^{3}} \frac{d^{3} \omega_{1}(\chi q)}{d q^{3}} \\
+\frac{5 \chi \Phi_{1}\left(\Phi_{1}-1\right)\left(\Phi_{1}-2\right)\left(\Phi_{1}-3\right)}{q^{4}} \frac{d^{2} \omega_{1}(\chi q)}{d q^{2}}+\frac{\Phi_{1}\left(\Phi_{1}-1\right)\left(\Phi_{1}-2\right)\left(\Phi_{1}-3\right)\left(\Phi_{1}-4\right)}{q^{5}} \frac{d \omega_{1}(\chi q)}{d q} \\
+h_{1}(q) f_{1}\left(\omega_{1}, \omega_{2}\right)=k_{1}(q), \\
\chi^{5} \frac{d^{6} \omega_{2}(\chi q)}{d q^{6}}+\frac{5 \chi^{4} \Phi_{2}}{q} \frac{d^{5} \omega_{2}(\chi q)}{d q^{5}}+\frac{10 \chi^{3} \Phi_{2}\left(\Phi_{2}-1\right)}{q^{2}} \frac{d^{4} \omega_{2}(\chi q)}{d q^{4}}+\frac{10 \chi^{2} \Phi_{2}\left(\Phi_{2}-1\right)\left(\Phi_{2}-2\right)}{q^{3}} \frac{d^{3} \omega_{2}(\chi q)}{d q^{3}} \\
+\frac{5 \chi \Phi_{2}\left(\Phi_{2}-1\right)\left(\Phi_{2}-2\right)\left(\Phi_{2}-3\right)}{q^{4}} \frac{d^{2} \omega_{2}(\chi q)}{d q^{2}}+\frac{\Phi_{2}\left(\Phi_{2}-1\right)\left(\Phi_{2}-2\right)\left(\Phi_{2}-3\right)\left(\Phi_{2}-4\right)}{q^{5}} \frac{d \omega_{2}(\chi q)}{d q} \\
+h_{2}(q) f_{2}\left(\omega_{1}, \omega_{2}\right)=k_{2}(q),
\end{array}\right.
$$

The ICs of the above system are written as:

$$
\left\{\begin{array}{l}
\omega_{1}=a_{1}, \frac{d \omega_{1}}{d q}=\frac{d^{2} \omega_{1}}{d q^{2}}=\frac{d^{3} \omega_{1}}{d q^{3}}=\frac{d^{4} \omega_{1}}{d q^{4}}=\frac{d^{5} \omega_{1}}{d q^{5}}=0, \\
\omega_{2}=a_{2}, \frac{d \omega_{2}}{d q}=\frac{d^{2} \omega_{2}}{d q^{2}}=\frac{d^{3} \omega_{2}}{d q^{3}}=\frac{d^{4} \omega_{2}}{d q^{4}}=\frac{d^{5} \omega_{2}}{d q^{5}}=0,
\end{array} \text { at } q \rightarrow 0 .\right.
$$

The singularity arises at $q=0$ five times as $q=q^{2}=q^{3}=q^{4}=q^{5}=0$ with SFs $5 \chi^{4} \Phi_{1}$, $10 \chi^{3} \Phi_{1}\left(\Phi_{1}-1\right), 10 \chi^{2} \Phi_{1}\left(\Phi_{1}-1\right)\left(\Phi_{1}-2\right), \quad 5 \chi \Phi_{1}\left(\Phi_{1}-1\right)\left(\Phi_{1}-2\right)\left(\Phi_{1}-3\right) \quad$ and $\Phi_{1}\left(\Phi_{1}-1\right)\left(\Phi_{1}-2\right)\left(\Phi_{1}-3\right)\left(\Phi_{1}-4\right)$ for $\omega_{1}$, while for the $\omega_{2}$, the SFs are $5 \chi^{4} \Phi_{2}, 10 \chi^{3} \Phi_{2}\left(\Phi_{2}-1\right)$, $10 \chi^{2} \Phi_{2}\left(\Phi_{2}-1\right)\left(\Phi_{2}-2\right), 5 \chi \Phi_{2}\left(\Phi_{2}-1\right)\left(\Phi_{2}-2\right)\left(\Phi_{2}-3\right)$ and $\Phi_{2}\left(\Phi_{2}-1\right)\left(\Phi_{2}-2\right)\left(\Phi_{2}-3\right)\left(\Phi_{2}-4\right)$. For the $\Phi_{1}=\Phi_{2}=1$, the SFs is $5 \chi^{4}$ and the $3^{\text {rd }}, 4^{\text {th }}, 5^{\text {th }}$ and $6^{\text {th }}$ terms vanish. For the $\Phi_{1}=\Phi_{2}=2$ , the SFs are $10 \chi^{4}$ and $20 \chi^{3}$, while the $4^{\text {th }}, 5^{\text {th }}$ and $6^{\text {th }}$ terms vanish. Moreover, For the $\Phi_{1}=\Phi_{2}=3$ , the SFs are $15 \chi^{4}, 60 \chi^{3}$ and $60 \chi^{2}$, while $5^{\text {th }}$ and $6^{\text {th }}$ terms vanish. Likewise, For the $\Phi_{1}=\Phi_{2}=4$ , the SFs are $20 \chi^{4}, 120 \chi^{3}, 240 \chi^{2}$ and $120 \chi$ while the $6^{\text {th }}$ term vanishes.

Type 2: The updated mathematical form of Eq (3) with $x=4$ and $w=2$ is given as:

$$
\left\{\begin{array}{l}
q^{-\Phi_{1}} \frac{d^{4}}{d q^{4}}\left(q^{\Phi_{1}} \frac{d^{2}}{d q^{2}}\right) \omega_{1}(\chi q)+h_{1}(q) f_{1}\left(\omega_{1}, \omega_{2}\right)=k_{1}(q), \\
q^{-\Phi_{2}} \frac{d^{4}}{d q^{4}}\left(q^{\Phi_{2}} \frac{d^{2}}{d q^{2}}\right) \omega_{2}(\chi q)+h_{2}(q) f_{2}\left(\omega_{1}, \omega_{2}\right)=k_{2}(q),
\end{array}\right.
$$

Solving the above derivative as: 


$$
\left\{\begin{array}{l}
\frac{d^{4}}{d q^{4}}\left(q^{\Phi_{1}} \frac{d^{2}}{d q^{2}}\right) \omega_{1}(\chi q)=\chi^{4} q^{\Phi_{1}} \frac{d^{6} \omega_{1}(\chi q)}{d q^{6}}+4 \chi^{3} \Phi_{1} q^{\Phi_{1}-1} \frac{d^{5} \omega_{1}(\chi q)}{d q^{5}}+6 \chi^{2} \Phi_{1}\left(\Phi_{1}-1\right) q^{\Phi_{1}-2} \frac{d^{4} \omega_{1}(\chi q)}{d q^{4}} \\
+4 \chi \Phi_{1}\left(\Phi_{1}-1\right)\left(\Phi_{1}-2\right) q^{\Phi_{1}-3} \frac{d^{3} \omega_{1}(\chi q)}{d q^{3}}+\Phi_{1}\left(\Phi_{1}-1\right)\left(\Phi_{1}-2\right)\left(\Phi_{1}-3\right) q^{\Phi_{1}-4} \frac{d^{2} \omega_{1}(\chi q)}{d q^{2}}, \\
\frac{d^{4}}{d q^{4}}\left(q^{\Phi_{2}} \frac{d^{2}}{d q^{2}}\right) \omega_{2}(\chi q)=\chi^{4} q^{\Phi_{2}} \frac{d^{6} \omega_{2}(\chi q)}{d q^{6}}+4 \chi^{3} \Phi_{2} q^{\Phi_{2}-1} \frac{d^{5} \omega_{2}(\chi q)}{d q^{5}}+6 \chi^{2} \Phi_{2}\left(\Phi_{2}-1\right) q^{\Phi_{2}-2} \frac{d^{4} \omega_{2}(\chi q)}{d q^{4}} \\
+4 \chi \Phi_{2}\left(\Phi_{2}-1\right)\left(\Phi_{2}-2\right) q^{\Phi_{2}-3} \frac{d^{3} \omega_{2}(\chi q)}{d q^{3}}+\Phi_{2}\left(\Phi_{2}-1\right)\left(\Phi_{2}-2\right)\left(\Phi_{2}-3\right) q^{\Phi_{2}-4} \frac{d^{2} \omega_{2}(\chi q)}{d q^{2}},
\end{array}\right.
$$

By using Eqs (14) and (15), the updated form of the nonlinear novel SSO-PDS is given as:

$$
\left\{\begin{array}{l}
\chi^{4} \frac{d^{6} \omega_{1}(\chi q)}{d q^{6}}+\frac{4 \chi^{3} \Phi_{1}}{q} \frac{d^{5} \omega_{1}(\chi q)}{d q^{5}}+\frac{6 \chi^{2} \Phi_{1}\left(\Phi_{1}-1\right)}{q^{2}} \frac{d^{4} \omega_{1}(\chi q)}{d q^{4}}+\frac{4 \chi \Phi_{1}\left(\Phi_{1}-1\right)\left(\Phi_{1}-2\right)}{q^{3}} \frac{d^{3} \omega_{1}(\chi q)}{d q^{3}} \\
+\frac{\Phi_{1}\left(\Phi_{1}-1\right)\left(\Phi_{1}-2\right)\left(\Phi_{1}-3\right)}{q^{4}} \frac{d^{2} \omega_{1}(\chi q)}{d q^{2}}+h_{1}(q) f_{1}\left(\omega_{1}, \omega_{2}\right)=k_{1}(q), \\
\chi^{4} \frac{d^{6} \omega_{2}(\chi q)}{d q^{6}}+\frac{4 \chi^{3} \Phi_{2}}{q} \frac{d^{5} \omega_{2}(\chi q)}{d q^{5}}+\frac{6 \chi^{2} \Phi_{2}\left(\Phi_{2}-1\right)}{q^{2}} \frac{d^{4} \omega_{2}(\chi q)}{d q^{4}}+\frac{4 \chi \Phi_{2}\left(\Phi_{2}-1\right)\left(\Phi_{2}-2\right)}{q^{3}} \frac{d^{3} \omega_{2}(\chi q)}{d q^{3}} \\
+\frac{\Phi_{2}\left(\Phi_{2}-1\right)\left(\Phi_{2}-2\right)\left(\Phi_{2}-3\right)}{q^{4}} \frac{d^{2} \omega_{2}(\chi q)}{d q^{2}}+h_{2}(q) f_{2}\left(\omega_{1}, \omega_{2}\right)=k_{2}(q) .
\end{array}\right.
$$

The ICs of the above system are written as:

$$
\left\{\begin{array}{l}
\omega_{1}=a_{1}, \frac{d \omega_{1}}{d q}=a_{3}, \frac{d^{2} \omega_{1}}{d q^{2}}=\frac{d^{3} \omega_{1}}{d q^{3}}=\frac{d^{4} \omega_{1}}{d q^{4}}=\frac{d^{5} \omega_{1}}{d q^{5}}=0, \\
\omega_{2}=a_{2}, \frac{d \omega_{2}}{d q}=a_{4}, \frac{d^{2} \omega_{2}}{d q^{2}}=\frac{d^{3} \omega_{2}}{d q^{3}}=\frac{d^{4} \omega_{2}}{d q^{4}}=\frac{d^{5} \omega_{2}}{d q^{5}}=0,
\end{array} \text { at } q \rightarrow 0 .\right.
$$

The singularity arises at $q=0$ four times as $q=q^{2}=q^{3}=q^{4}=0$ with SFs $4 \chi^{3} \Phi_{1}, 6 \chi^{2} \Phi_{1}\left(\Phi_{1}-1\right)$, $4 \chi \Phi_{1}\left(\Phi_{1}-1\right)\left(\Phi_{1}-2\right)$ and $\Phi_{1}\left(\Phi_{1}-1\right)\left(\Phi_{1}-2\right)\left(\Phi_{1}-3\right)$ for $\omega_{1}$, while for the $\omega_{2}$, the SFs are $4 \chi^{3} \Phi_{2}$ , $6 \chi^{2} \Phi_{2}\left(\Phi_{2}-1\right), 4 \chi \Phi_{2}\left(\Phi_{2}-1\right)\left(\Phi_{2}-2\right)$ and $\Phi_{2}\left(\Phi_{2}-1\right)\left(\Phi_{2}-2\right)\left(\Phi_{2}-3\right)$. For $\Phi_{1}=\Phi_{2}=1$, the SF is $4 \chi^{3}$ and the $3^{\text {rd }}, 4^{\text {th }}$ and $5^{\text {th }}$ terms vanish. For the $\Phi_{1}=\Phi_{2}=2$, the SFs are $8 \chi^{3}$ and $12 \chi^{2}$, while the $4^{\text {th }}$ and $5^{\text {th }}$ terms vanish. Moreover, For the $\Phi_{1}=\Phi_{2}=3$, the SFs are $12 \chi^{3}, 36 \chi^{2}$ and $24 \chi$, while the $5^{\text {th }}$ term vanishes.

Type 3: The updated mathematical form of Eq (3) with $x=3$ and $w=3$ is given as: 


$$
\left\{\begin{array}{l}
q^{-\Phi_{1}} \frac{d^{3}}{d q^{3}}\left(q^{\Phi_{1}} \frac{d^{3}}{d q^{3}}\right) \omega_{1}(\chi q)+h_{1}(q) f_{1}\left(\omega_{1}, \omega_{2}\right)=k_{1}(q), \\
q^{-\Phi_{2}} \frac{d^{3}}{d q^{3}}\left(q^{\Phi_{2}} \frac{d^{3}}{d q^{3}}\right) \omega_{2}(\chi q)+h_{2}(q) f_{2}\left(\omega_{1}, \omega_{2}\right)=k_{2}(q),
\end{array}\right.
$$

Solving the above derivative as:

$$
\left\{\begin{array}{l}
\frac{d^{3}}{d q^{3}}\left(q^{\Phi_{1}} \frac{d^{3}}{d q^{3}}\right) \omega_{1}(\chi q)=\chi^{3} q^{\Phi_{1}} \frac{d^{6} \omega_{1}(\chi q)}{d q^{6}}+3 \chi^{2} \Phi_{1} q^{\Phi_{1}-1} \frac{d^{5} \omega_{1}(\chi q)}{d q^{5}}+3 \chi \Phi_{1}\left(\Phi_{1}-1\right) q^{\Phi_{1}-2} \frac{d^{4} \omega_{1}(\chi q)}{d q^{4}} \\
+\Phi_{1}\left(\Phi_{1}-1\right)\left(\Phi_{1}-2\right) q^{\Phi_{1}-3} \frac{d^{3} \omega_{1}(\chi q)}{d q^{3}} \\
\frac{d^{3}}{d q^{3}}\left(q^{\Phi_{2}} \frac{d^{3}}{d q^{3}}\right) \omega_{2}(\chi q)=\chi^{3} q^{\Phi_{2}} \frac{d^{6} \omega_{2}(\chi q)}{d q^{6}}+3 \chi^{2} \Phi_{2} q^{\Phi_{2}-1} \frac{d^{5} \omega_{2}(\chi q)}{d q^{5}}+3 \chi \Phi_{2}\left(\Phi_{2}-1\right) q^{\Phi_{2}-2} \frac{d^{4} \omega_{2}(\chi q)}{d q^{4}} \\
+\Phi_{2}\left(\Phi_{2}-1\right)\left(\Phi_{2}-2\right) q^{\Phi_{2}-3} \frac{d^{3} \omega_{2}(\chi q)}{d q^{3}} .
\end{array}\right.
$$

By using Eqs (18) and (19), the updated form of the nonlinear novel SSO-PDS is given as:

$$
\left\{\begin{array}{l}
\chi^{3} \frac{d^{6} \omega_{1}(\chi q)}{d q^{6}}+\frac{3 \chi^{2} \Phi_{1}}{q} \frac{d^{5} \omega_{1}(\chi q)}{d q^{5}}+\frac{3 \chi \Phi_{1}\left(\Phi_{1}-1\right)}{q^{2}} \frac{d^{4} \omega_{1}(\chi q)}{d q^{4}}+\frac{\Phi_{1}\left(\Phi_{1}-1\right)\left(\Phi_{1}-2\right)}{q^{3}} \frac{d^{3} \omega_{1}(\chi q)}{d q^{3}} \\
+h_{1}(q) f_{1}\left(\omega_{1}, \omega_{2}\right)=k_{1}(q), \\
\chi^{3} \frac{d^{6} \omega_{2}(\chi q)}{d q^{6}}+\frac{3 \chi^{2} \Phi_{2}}{q} \frac{d^{5} \omega_{2}(\chi q)}{d q^{5}}+\frac{3 \chi \Phi_{2}\left(\Phi_{2}-1\right)}{q^{2}} \frac{d^{4} \omega_{2}(\chi q)}{d q^{4}}+\frac{\Phi_{2}\left(\Phi_{2}-1\right)\left(\Phi_{2}-2\right)}{q^{3}} \frac{d^{3} \omega_{2}(\chi q)}{d q^{3}} \\
+h_{2}(q) f_{2}\left(\omega_{1}, \omega_{2}\right)=k_{2}(q) .
\end{array}\right.
$$

The ICs of the above system are written as:

$$
\left\{\begin{array}{l}
\omega_{1}=a_{1}, \frac{d \omega_{1}}{d q}=a_{3}, \frac{d^{2} \omega_{1}}{d q^{2}}=a_{5}, \frac{d^{3} \omega_{1}}{d q^{3}}=\frac{d^{4} \omega_{1}}{d q^{4}}=\frac{d^{5} \omega_{1}}{d q^{5}}=0, \\
\omega_{2}=a_{2}, \frac{d \omega_{2}}{d q}=a_{4}, \frac{d^{2} \omega_{2}}{d q^{2}}=a_{6}, \frac{d^{3} \omega_{2}}{d q^{3}}=\frac{d^{4} \omega_{2}}{d q^{4}}=\frac{d^{5} \omega_{2}}{d q^{5}}=0,
\end{array} \text { at } q \rightarrow 0 .\right.
$$

The singularity arises at $q=0$ three times as $q=q^{2}=q^{3}=0$ with SFs $3 \chi^{2} \Phi_{1}, 3 \chi \Phi_{1}\left(\Phi_{1}-1\right)$ and $\Phi_{1}\left(\Phi_{1}-1\right)\left(\Phi_{1}-2\right)$ for $\omega_{1}$, while for the $\omega_{2}$, the SFs are $3 \chi^{2} \Phi_{2}, 3 \chi \Phi_{2}\left(\Phi_{2}-1\right)$ and $\Phi_{2}\left(\Phi_{2}-1\right)\left(\Phi_{2}-2\right)$. For $\Phi_{1}=\Phi_{2}=1$, the SF is $3 \chi^{2}$ and the $3^{\text {rd }}$ and $4^{\text {th }}$ terms vanish. For the $\Phi_{1}=\Phi_{2}=2$, the SFs are $6 \chi^{2}$ and $6 \chi$, while the $4^{\text {th }}$ term vanishes.

Type 4: The updated mathematical form of Eq (3) with $x=4$ and $w=2$ is given as: 


$$
\left\{\begin{array}{l}
q^{-\Phi_{1}} \frac{d^{2}}{d q^{2}}\left(q^{\Phi_{1}} \frac{d^{4}}{d q^{4}}\right) \omega_{1}(\chi q)+h_{1}(q) f_{1}\left(\omega_{1}, \omega_{2}\right)=k_{1}(q), \\
q^{-\Phi_{2}} \frac{d^{2}}{d q^{2}}\left(q^{\Phi_{2}} \frac{d^{4}}{d q^{4}}\right) \omega_{2}(\chi q)+h_{2}(q) f_{2}\left(\omega_{1}, \omega_{2}\right)=k_{2}(q),
\end{array}\right.
$$

Solving the above derivative as:

$$
\left\{\begin{array}{l}
\frac{d^{2}}{d q^{2}}\left(q^{\Phi_{1}} \frac{d^{4}}{d q^{4}}\right) \omega_{1}(\chi q)=\chi^{2} q^{\Phi_{1}} \frac{d^{6} \omega_{1}(\chi q)}{d q^{6}}+2 \chi \Phi_{1} q^{\Phi_{1}-1} \frac{d^{5} \omega_{1}(\chi q)}{d q^{5}}+\Phi_{1}\left(\Phi_{1}-1\right) q^{\Phi_{1}-2} \frac{d^{4} \omega_{1}(\chi q)}{d q^{4}}, \\
\frac{d^{2}}{d q^{2}}\left(q^{\Phi_{2}} \frac{d^{4}}{d q^{4}}\right) \omega_{2}(\chi q)=\chi^{2} q^{\Phi_{2}} \frac{d^{6} \omega_{2}(\chi q)}{d q^{6}}+2 \chi \Phi_{2} q^{\Phi_{2}-1} \frac{d^{5} \omega_{2}(\chi q)}{d q^{5}}+\Phi_{2}\left(\Phi_{2}-1\right) q^{\Phi_{2}-2} \frac{d^{4} \omega_{2}(\chi q)}{d q^{4}} .
\end{array}\right.
$$

By using Eqs (22) and (23), the updated form of the nonlinear novel SSO-PDS is given as:

$$
\left\{\begin{array}{l}
\chi^{2} \frac{d^{6} \omega_{1}(\chi q)}{d q^{6}}+\frac{2 \chi \Phi_{1}}{q} \frac{d^{5} \omega_{1}(\chi q)}{d q^{5}}+\frac{\Phi_{1}\left(\Phi_{1}-1\right)}{q^{2}} \frac{d^{4} \omega_{1}(\chi q)}{d q^{4}}+h_{1}(q) f_{1}\left(\omega_{1}, \omega_{2}\right)=k_{1}(q), \\
\chi^{2} \frac{d^{6} \omega_{2}(\chi q)}{d q^{6}}+\frac{2 \chi \Phi_{2}}{q} \frac{d^{5} \omega_{2}(\chi q)}{d q^{5}}+\frac{\Phi_{2}\left(\Phi_{2}-1\right)}{q^{2}} \frac{d^{4} \omega_{2}(\chi q)}{d q^{4}}+h_{2}(q) f_{2}\left(\omega_{1}, \omega_{2}\right)=k_{2}(q) .
\end{array}\right.
$$

The ICs of the above system are written as:

$$
\left\{\begin{array}{l}
\omega_{1}=a_{1}, \frac{d \omega_{1}}{d q}=a_{3}, \frac{d^{2} \omega_{1}}{d q^{2}}=a_{5}, \frac{d^{3} \omega_{1}}{d q^{3}}=a_{7}, \frac{d^{4} \omega_{1}}{d q^{4}}=\frac{d^{5} \omega_{1}}{d q^{5}}=0, \\
\omega_{2}=a_{2}, \frac{d \omega_{2}}{d q}=a_{4}, \frac{d^{2} \omega_{2}}{d q^{2}}=a_{6}, \frac{d^{3} \omega_{2}}{d q^{3}}=a_{8}, \frac{d^{4} \omega_{2}}{d q^{4}}=\frac{d^{5} \omega_{2}}{d q^{5}}=0,
\end{array} \text { at } q \rightarrow 0 .\right.
$$

The singularity arises at $q=0$ twice as $q=q^{2}=0$ with SFs $2 \chi \Phi_{1}$ and $\Phi_{1}\left(\Phi_{1}-1\right)$ for $\omega_{1}$, while for the $\omega_{2}$, the SFs are $2 \chi \Phi_{2}$ and $\Phi_{2}\left(\Phi_{2}-1\right)$. For $\Phi_{1}=\Phi_{2}=1$, the SF is $2 \chi$ and the $3^{\text {rd }}$ term vanishes. Type 5: The updated mathematical form of Eq (3) with $x=1$ and $w=5$ is given as:

$$
\left\{\begin{array}{l}
q^{-\Phi_{1}} \frac{d}{d q}\left(q^{\Phi_{1}} \frac{d^{5}}{d q^{5}}\right) \omega_{1}(\chi q)+h_{1}(q) f_{1}\left(\omega_{1}, \omega_{2}\right)=k_{1}(q), \\
q^{-\Phi_{2}} \frac{d}{d q}\left(q^{\Phi_{2}} \frac{d^{5}}{d q^{5}}\right) \omega_{2}(\chi q)+h_{2}(q) f_{2}\left(\omega_{1}, \omega_{2}\right)=k_{2}(q),
\end{array}\right.
$$

Solving the above derivative as: 


$$
\left\{\begin{array}{l}
\frac{d}{d q}\left(q^{\Phi_{1}} \frac{d^{5}}{d q^{5}}\right) \omega_{1}(\chi q)=\chi q^{\Phi_{1}} \frac{d^{6} \omega_{1}(\chi q)}{d q^{6}}+\Phi_{1} q^{\Phi_{1}-1} \frac{d^{5} \omega_{1}(\chi q)}{d q^{5}} \\
\frac{d}{d q}\left(q^{\Phi_{2}} \frac{d^{5}}{d q^{5}}\right) \omega_{2}(\chi q)=\chi q^{\Phi_{2}} \frac{d^{6} \omega_{2}(\chi q)}{d q^{6}}+\Phi_{2} q^{\Phi_{2}-1} \frac{d^{5} \omega_{2}(\chi q)}{d q^{5}} .
\end{array}\right.
$$

By using Eqs (26) and (27), the updated form of the nonlinear novel SSO-PDS is given as:

$$
\left\{\begin{array}{l}
\chi \frac{d^{6} \omega_{1}(\chi q)}{d q^{6}}+\frac{\Phi_{1}}{q} \frac{d^{5} \omega_{1}(\chi q)}{d q^{5}}+h_{1}(q) f_{1}\left(\omega_{1}, \omega_{2}\right)=k_{1}(q) \\
\chi \frac{d^{6} \omega_{2}(\chi q)}{d q^{6}}+\frac{\Phi_{2}}{q} \frac{d^{5} \omega_{2}(\chi q)}{d q^{5}}+h_{2}(q) f_{2}\left(\omega_{1}, \omega_{2}\right)=k_{2}(q) .
\end{array}\right.
$$

The ICs of the above system are written as:

$$
\begin{cases}\omega_{1}=a_{1}, \frac{d \omega_{1}}{d q}=a_{3}, \frac{d^{2} \omega_{1}}{d q^{2}}=a_{5}, \frac{d^{3} \omega_{1}}{d q^{3}}=a_{7}, \frac{d^{4} \omega_{1}}{d q^{4}}=a_{9}, \frac{d^{5} \omega_{1}}{d q^{5}}=0, & \text { at } q \rightarrow 0 . \\ \omega_{2}=a_{2}, \frac{d \omega_{2}}{d q}=a_{4}, \frac{d^{2} \omega_{2}}{d q^{2}}=a_{6}, \frac{d^{3} \omega_{2}}{d q^{3}}=a_{8}, \frac{d^{4} \omega_{2}}{d q^{4}}=a_{10}, \frac{d^{5} \omega_{2}}{d q^{5}}=0, & \end{cases}
$$

The singularity arises at $q=0$ single time as $q=0$ with SF $\Phi_{1}$ for $\omega_{1}$, while for the $\omega_{2}$, the SF is $\Phi_{2}$.

\section{Methodology}

In this section, the solution of each example of the nonlinear novel SSO-PDS using the designed ANNs-LMB in two phases. First, an essential detail is provided based on the designed stochastic ANNs-LMB. Whereas, an execution procedure for the designed ANNs-LMB are provided in the second phase. The workflow diagram of the designed stochastic ANNs-LMB is drawn in Fig. 1. The reference solutions are provided to authenticate the exactness of those results, which have been obtained through the designed stochastic ANNs-LMB. Fig 2 provides the information of a single neuron in the ANNs, where the ANNs-LMB is implemented using the 'nftool' in the "Matlab" based on the testing, authentication and training statistics, appropriate hidden neuron settings together with the designed methodology. 


\section{Methodology}

Reference solutions

Designed dataset to solve the nonlinear novel SSO-PDS along with its five categories

\section{Intelligent computing}

Multi-layer process using the designed ANNsLMB

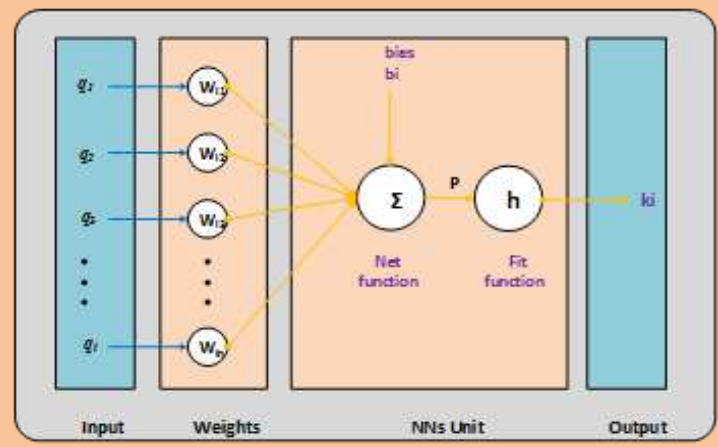

Neuron Model

\section{Results Simulations}

Comparison of the results and $\mathrm{AE}$ for each case category of the nonlinear novel SSO-PDS
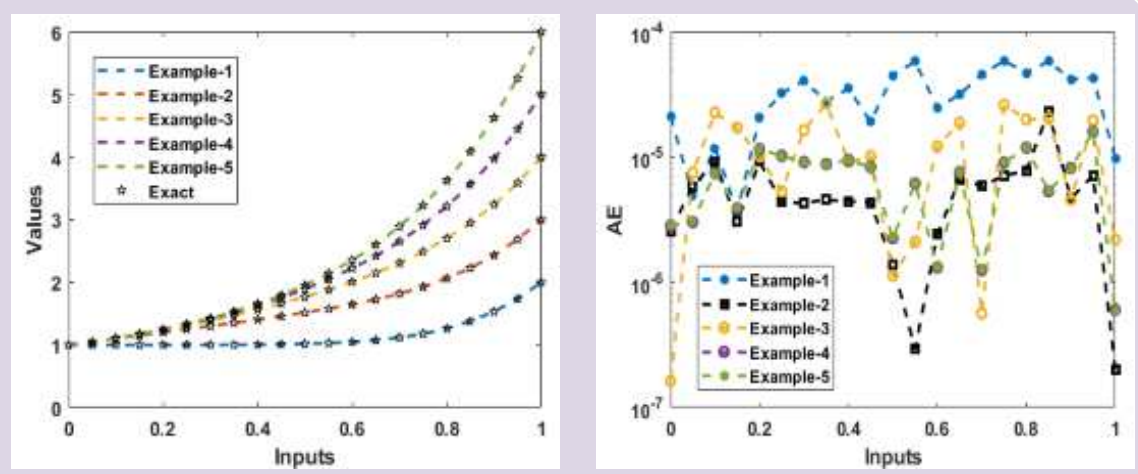

Result Comparison

$\mathrm{AE}$

Proposed solutions through ANNs-LMB using the M.S.E, Fit, Regression plots, EHs and State transition for the nonlinear novel SSO-PDS along with its five categories

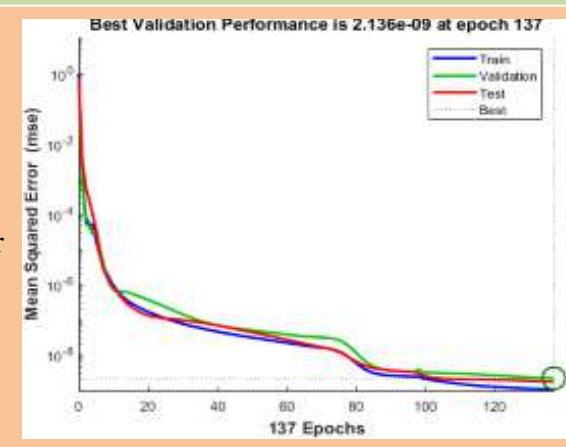

Performance

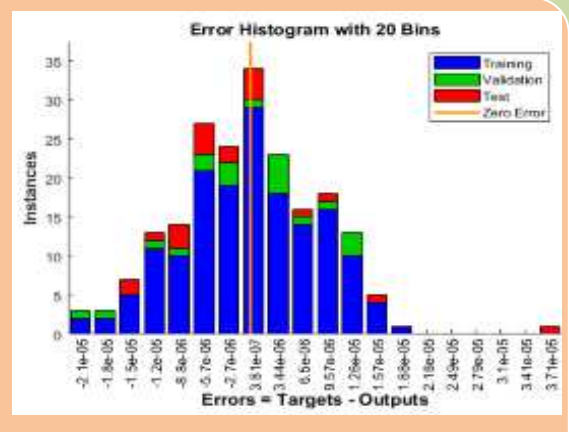

EHS

Fig 1: Workflow diagram based on the ANNs-LMB to solve the nonlinear novel SSO-PDS 


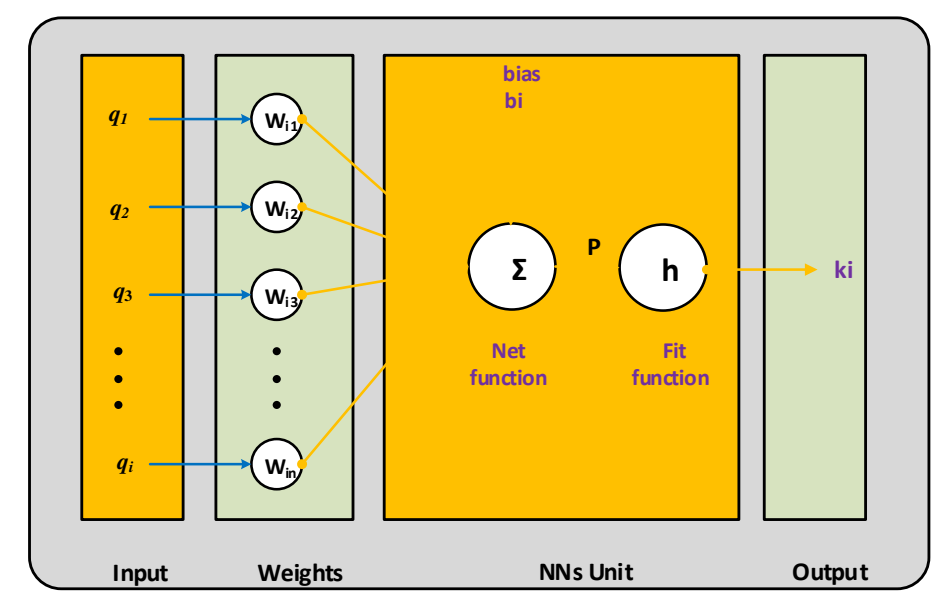

Fig 2: Proposed network based single neuron

\section{Results and discussions}

In this section, the numerical solutions of all types with one example based on the nonlinear novel SSO-PDS are presented using the designed ANNs-LMB. The numerical discussions are provided on the basis of ANNs-LMB and the numerical outcomes are calculated in input $[0,1]$ with the step size of 0.01 for solving the nonlinear novel SSO-PDS. The proposed ANNs-LMB is applied to solve one variant of each category of the nonlinear novel SSO-PDS using the "nftool' in "Matlab" with 12 hidden numbers of neurons. The training, validation and testing data have been using $80 \%$, $10 \%$ and $10 \%$ of the optimization procedures of ANNs-LMB. The proposed ANNs-LMB is provided in Fig 3, whereas, the ANNs-LMB is proficient to solve each category of the nonlinear novel SSO-PDS.

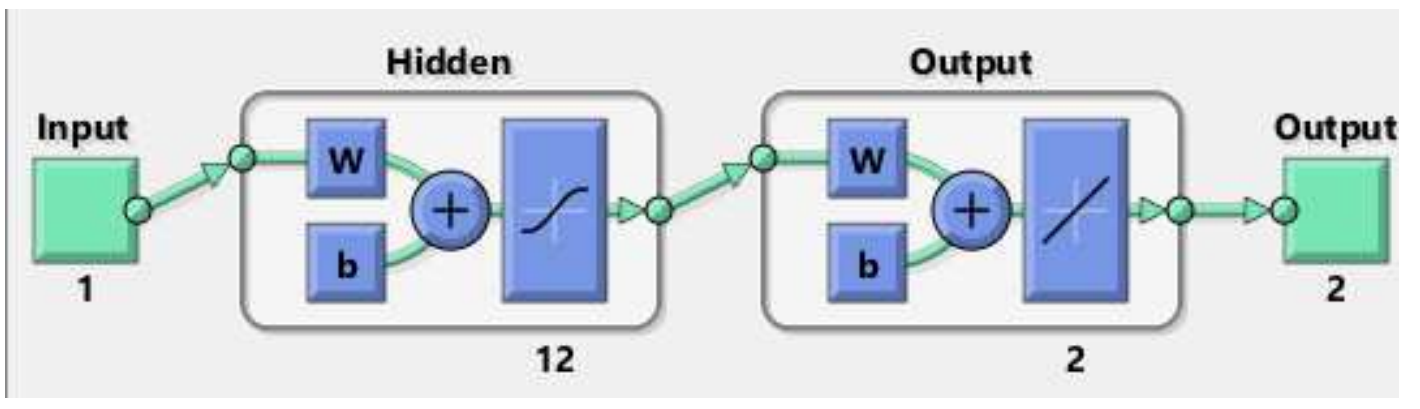

Fig 3: Designed measures of the ANNs-LMB for solving the nonlinear novel SSO-PDS

\subsection{Numerical performances of the SSO-LMB}

This section presents the solutions of all categories-based examples for solving the nonlinear novel SSO-PDS using the stochastic measures ANNs-LMB. These estimated nonlinear singular systems are derived in the below examples.

Example 1: Consider a nonlinear novel SSO-PDS by taking the values of $\chi=\frac{1}{2}$ and $\omega_{1}=\omega_{2}=5$ in Eqs (12) and (13) is given as: 


$$
\begin{aligned}
& \left\{\begin{array}{l}
\frac{1}{32} \frac{d^{6} \omega_{1}}{d q^{6}}\left(\frac{1}{2} q\right)+\frac{25}{16 q} \frac{d^{5} \omega_{1}}{d q^{5}}\left(\frac{1}{2} q\right)+\frac{25}{q^{2}} \frac{d^{4} \omega_{1}}{d q^{4}}\left(\frac{1}{2} q\right)+\frac{150}{q^{3}} \frac{d^{3} \omega_{1}}{d q^{3}}\left(\frac{1}{2} q\right)+\frac{300}{q^{4}} \frac{d^{2} \omega_{1}}{d q^{2}}\left(\frac{1}{2} q\right) \\
+\frac{120}{q^{5}} \frac{d \omega_{1}}{d q}\left(\frac{1}{2} q\right)+\omega_{1} \omega_{2}=5671-q^{12} \\
\frac{1}{32} \frac{d^{6} \omega_{2}}{d q^{6}}\left(\frac{1}{2} q\right)+\frac{25}{16 q} \frac{d^{5} \omega_{2}}{d q^{5}}\left(\frac{1}{2} q\right)+\frac{25}{q^{2}} \frac{d^{4} \omega_{2}}{d q^{4}}\left(\frac{1}{2} q\right)+\frac{150}{q^{3}} \frac{d^{3} \omega_{2}}{d q^{3}}\left(\frac{1}{2} q\right)+\frac{300}{q^{4}} \frac{d^{2} \omega_{2}}{d q^{2}}\left(\frac{1}{2} q\right) \\
+\frac{120}{q^{5}} \frac{d \omega_{2}}{d q}\left(\frac{1}{2} q\right)-\omega_{1} \omega_{2}=-5671+q^{12} . \\
\omega_{1}=1, \frac{d \omega_{1}}{d q}=\frac{d^{2} \omega_{1}}{d q^{2}}=\frac{d^{3} \omega_{1}}{d q^{3}}=\frac{d^{4} \omega_{1}}{d q^{4}}=\frac{d^{5} \omega_{1}}{d q^{5}}=0, \\
\omega_{2}=1, \frac{d \omega_{2}}{d q}=\frac{d^{2} \omega_{2}}{d q^{2}}=\frac{d^{3} \omega_{2}}{d q^{3}}=\frac{d^{4} \omega_{2}}{d q^{4}}=\frac{d^{5} \omega_{2}}{d q^{5}}=0,
\end{array}\right.
\end{aligned}
$$

The true form of the solution is $\left[1+q^{6}, 1-q^{6}\right]$.

Example 2: Consider a nonlinear novel SSO-PDS by taking the values of $\chi=\frac{1}{2}$ and $\omega_{1}=\omega_{2}=4$ in Eqs (16) and (17) is given as:

$$
\begin{aligned}
& \left\{\begin{array}{l}
\frac{1}{16} \frac{d^{6} \omega_{1}}{d q^{6}}\left(\frac{1}{2} q\right)+\frac{2}{q} \frac{d^{5} \omega_{1}}{d q^{5}}\left(\frac{1}{2} q\right)+\frac{18}{q^{2}} \frac{d^{4} \omega_{1}}{d q^{4}}\left(\frac{1}{2} q\right)+\frac{48}{q^{3}} \frac{d^{3} \omega_{1}}{d q^{3}}\left(\frac{1}{2} q\right)+\frac{24}{q^{4}} \frac{d^{2} \omega_{1}}{d q^{2}}\left(\frac{1}{2} q\right)+\omega_{1} \omega_{2}=3151+2 q+q^{2}-q^{12}, \\
\frac{1}{16} \frac{d^{6} \omega_{2}}{d q^{6}}\left(\frac{1}{2} q\right)+\frac{2}{q} \frac{d^{5} \omega_{2}}{d q^{5}}\left(\frac{1}{2} q\right)+\frac{18}{q^{2}} \frac{d^{4} \omega_{2}}{d q^{4}}\left(\frac{1}{2} q\right)+\frac{48}{q^{3}} \frac{d^{3} \omega_{2}}{d q^{3}}\left(\frac{1}{2} q\right)+\frac{24}{q^{4}} \frac{d^{2} \omega_{2}}{d q^{2}}\left(\frac{1}{2} q\right)-\omega_{1} \omega_{2}=-3151-2 q-q^{2}+q^{12}
\end{array}\right. \\
& \left\{\begin{array}{l}
\omega_{1}=\frac{d \omega_{1}}{d q}=1, \frac{d^{2} \omega_{1}}{d q^{2}}=\frac{d^{3} \omega_{1}}{d q^{3}}=\frac{d^{4} \omega_{1}}{d q^{4}}=\frac{d^{5} \omega_{1}}{d q^{5}}=0, \quad \text { at } q \rightarrow 0 . \\
\omega_{2}=\frac{d \omega_{2}}{d q}=1, \frac{d^{2} \omega_{2}}{d q^{2}}=\frac{d^{3} \omega_{2}}{d q^{3}}=\frac{d^{4} \omega_{2}}{d q^{4}}=\frac{d^{5} \omega_{2}}{d q^{5}}=0, \quad
\end{array}\right.
\end{aligned}
$$

The true form of the solution is $\left[1+q+q^{6}, 1+q-q^{6}\right]$.

Example 3: Consider a nonlinear novel SSO-PDS by taking the values of $\chi=\frac{1}{2}$ and $\omega_{1}=\omega_{2}=3$ in Eqs (20) and (21) is given as: 


$$
\begin{aligned}
& \left\{\frac{1}{8} \frac{d^{6} \omega_{1}}{d q^{6}}\left(\frac{1}{2} q\right)+\frac{9}{4 q} \frac{d^{5} \omega_{1}}{d q^{5}}\left(\frac{1}{2} q\right)+\frac{9}{q^{2}} \frac{d^{4} \omega_{1}}{d q^{4}}\left(\frac{1}{2} q\right)+\frac{6}{q^{3}} \frac{d^{3} \omega_{1}}{d q^{3}}\left(\frac{1}{2} q\right)+\omega_{1} \omega_{2}=1801+2 q+3 q^{2}+2 q^{3}+q^{4}-q^{12},\right. \\
& \left\{\frac{1}{8} \frac{d^{6} \omega_{2}}{d q^{6}}\left(\frac{1}{2} q\right)+\frac{9}{4 q} \frac{d^{5} \omega_{2}}{d q^{5}}\left(\frac{1}{2} q\right)+\frac{9}{q^{2}} \frac{d^{4} \omega_{2}}{d q^{4}}\left(\frac{1}{2} q\right)+\frac{6}{q^{3}} \frac{d^{3} \omega_{2}}{d q^{3}}\left(\frac{1}{2} q\right)-\omega_{1} \omega_{2}=-1801-2 q-3 q^{2}-2 q^{3}-q^{4}+q^{12}\right. \text {. } \\
& \left\{\begin{array}{l}
\omega_{1}=\frac{d \omega_{1}}{d q}=1, \frac{d^{2} \omega_{1}}{d q^{2}}=2, \frac{d^{3} \omega_{1}}{d q^{3}}=\frac{d^{4} \omega_{1}}{d q^{4}}=\frac{d^{5} \omega_{1}}{d q^{5}}=0, \\
\omega_{2}=\frac{d \omega_{2}}{d q}=1, \frac{d^{2} \omega_{2}}{d q^{2}}=2, \frac{d^{3} \omega_{2}}{d q^{3}}=\frac{d^{4} \omega_{2}}{d q^{4}}=\frac{d^{5} \omega_{2}}{d q^{5}}=0,
\end{array} \text { at } q \rightarrow 0 .\right.
\end{aligned}
$$

The true form of the solution is $\left[1+q+q^{2}+q^{6}, 1+q+q^{2}-q^{6}\right]$

Example 4: Consider a nonlinear novel SSO-PDS by taking the values of $\chi=\frac{1}{2}$ and $\omega_{1}=\omega_{2}=2$ in Eqs (24) and (25) is given as:

$$
\begin{aligned}
& \left\{\begin{array}{l}
\frac{1}{4} \frac{d^{6} \omega_{1}}{d q^{6}}\left(\frac{1}{2} q\right)+\frac{2}{q} \frac{d^{5} \omega_{1}}{d q^{5}}\left(\frac{1}{2} q\right)+\frac{2}{q^{2}} \frac{d^{4} \omega_{1}}{d q^{4}}\left(\frac{1}{2} q\right)+\omega_{1} \omega_{2}=1081+2 q+3 q^{2}+4 q^{3}+3 q^{4}+2 q^{5}+q^{6}-q^{12}, \\
\frac{1}{4} \frac{d^{6} \omega_{2}}{d q^{6}}\left(\frac{1}{2} q\right)+\frac{2}{q} \frac{d^{5} \omega_{2}}{d q^{5}}\left(\frac{1}{2} q\right)+\frac{2}{q^{2}} \frac{d^{4} \omega_{2}}{d q^{4}}\left(\frac{1}{2} q\right)-\omega_{1} \omega_{2}=-1081-2 q-3 q^{2}-4 q^{3}-3 q^{4}-2 q^{5}-q^{6}+q^{12}
\end{array}\right. \\
& \left\{\begin{array}{l}
\omega_{1}=\frac{d \omega_{1}}{d q}=1, \frac{d^{2} \omega_{1}}{d q^{2}}=2, \frac{d^{3} \omega_{1}}{d q^{3}}=6, \frac{d^{4} \omega_{1}}{d q^{4}}=\frac{d^{5} \omega_{1}}{d q^{5}}=0, \\
\omega_{2}=\frac{d \omega_{2}}{d q}=1, \frac{d^{2} \omega_{2}}{d q^{2}}=2, \frac{d^{3} \omega_{2}}{d q^{3}}=6, \frac{d^{4} \omega_{2}}{d q^{4}}=\frac{d^{5} \omega_{2}}{d q^{5}}=0,
\end{array} \text { at } q \rightarrow 0 .\right.
\end{aligned}
$$

The true form of the solution is $\left[1+q+q^{2}+q^{3}+q^{6}, 1+q+q^{2}+q^{3}-q^{6}\right]$

Example 5: Consider a nonlinear novel SSO-PDS by taking the values of $\chi=\frac{1}{2}$ and $\omega_{1}=\omega_{2}=1$ in Eqs (28) and (29) is given as:

$$
\left\{\begin{array}{l}
\frac{1}{2} \frac{d^{6} \omega_{1}}{d q^{6}}\left(\frac{1}{2} q\right)+\frac{2}{q} \frac{d^{5} \omega_{1}}{d q^{5}}\left(\frac{1}{2} q\right)+\omega_{1} \omega_{2}=1081+2 q+3 q^{2}+4 q^{3}+5 q^{4}+4 q^{5}+3 q^{6}+2 q^{7}+q^{8}-q^{12} \\
\frac{1}{2} \frac{d^{6} \omega_{2}}{d q^{6}}\left(\frac{1}{2} q\right)+\frac{2}{q} \frac{d^{5} \omega_{2}}{d q^{5}}\left(\frac{1}{2} q\right)-\omega_{1} \omega_{2}=-1081-2 q-3 q^{2}-4 q^{3}-5 q^{4}-4 q^{5}-3 q^{6}-2 q^{7}-q^{8}+q^{12} .
\end{array}\right.
$$




$$
\left\{\begin{array}{l}
\omega_{1}=\frac{d \omega_{1}}{d q}=1, \frac{d^{2} \omega_{1}}{d q^{2}}=2, \frac{d^{3} \omega_{1}}{d q^{3}}=6, \frac{d^{4} \omega_{1}}{d q^{4}}=24, \frac{d^{5} \omega_{1}}{d q^{5}}=0, \\
\omega_{2}=\frac{d \omega_{2}}{d q}=1, \frac{d^{2} \omega_{2}}{d q^{2}}=2, \frac{d^{3} \omega_{2}}{d q^{3}}=6, \frac{d^{4} \omega_{2}}{d q^{4}}=24, \frac{d^{5} \omega_{2}}{d q^{5}}=0,
\end{array} \text { at } q \rightarrow 0 .\right.
$$

The true form of the solution is $\left[1+q+q^{2}+q^{3}+q^{4}+q^{6}, 1+q+q^{2}+q^{3}+q^{4}-q^{6}\right]$

The plots of each class of the nonlinear novel SSO-PDS by using the designed ANNs-LMB is presented in Figs 4-8. The graphical results of the nonlinear novel SSO-PDS based examples of each class using the performance and transition states are drawn in Figs 4 and 5. The convergence routines through MSE using the testing, training, validation and best curve for each class of the nonlinear novel SSO-PDS by using the designed ANNs-LMB in presented in Fig 4. The best validation performance at epoch 136, 266, 490, 1000 and 1000 are found around $2.136 \times 10^{-09}$, $1.045 \times 10^{-08}, 2.480 \times 10^{-09}, 2.480 \times 10^{-09}$ and $6.570 \times 10^{-10}$, respectively. The gradient measures are demonstrated in Fig 5 using the step-size $(\mathrm{Mu})$ is measured through the ANNs-LMB for each class of nonlinear novel SSO-PDS $\left[9.793 \times 10^{-08}, 9.976 \times 10^{-08}, 9.967 \times 10^{-08}, 1.625 \times 10^{-07}\right.$ and $\left.1.461 \times 10^{-07}\right]$ and $\left[1 \times 10^{-09}, 1 \times 10^{-09}, 1 \times 10^{-08}, 1 \times 10^{-08}\right.$ and $\left.1 \times 10^{-08}\right]$. These compatible performances indicate the convergence and correctness of the proposed ANNs-LMB for each class of nonlinear novel SSOPDS.

Figs 6 validates the curve fitting values for each class of nonlinear novel SSO-PDS. These conspiracies specify the result comparisons based on the ANNs-LMB with the reference form of the solutions for each class of nonlinear novel SSO-PDS along with the error plots. The performances using the maximum error through the training, substantiation and testing intervals using the proposed ANNs-LMB calculated around $10^{-06}$ to $10^{-08}$ for each class of nonlinear novel SSO-PDS. The EHs performances are plotted in Fig 7 to examine the error inquiries using the output/input grids to solve each class of nonlinear novel SSO-PDS. The average values of the EHs with the position of zero-line calculated around $3.46 \times 10^{-06}, 1.84 \times 10^{-05}, 5.32 \times 10^{-06}, 2.02 \times 10^{-06}$ and $3.81 \times 10^{-07}$ for example 1, 2, 3, 4 and 5 of nonlinear novel SSO-PDS. The regressions values are drawn in Figs 8 for each class of nonlinear novel SSO-PDS. These graphical correlation performances are applied to calculate the regression performance. It is observed that the correlation (R) values calculated around 1 for each class of nonlinear novel SSO-PDS, which represent the perfect model based on the training, substantiation and testing. This mathematical presents the exactness of the ANNS-LMB for each class of nonlinear novel SSO-PDS. Furthermore, the MSE is capable for the training, substantiation and testing, backpropagation actions, complexity, time and epochs are provided in the below Table to solve the nonlinear novel SSO-PDS.

Table 1: ANNs-LMB results for each class of the nonlinear novel SSO-PDS

\begin{tabular}{|c|c|c|c|c|c|c|c|c|}
\hline \multirow{2}{*}{ Example } & \multicolumn{3}{|c|}{ MSE } & \multirow{2}{*}{ Performance } & \multirow{2}{*}{ Gradient } & \multirow{2}{*}{ Mu } & \multirow{2}{*}{ Epoch } & \multirow{2}{*}{ Time } \\
\hline & Training e & Verification & Testing & & & & & \\
\hline 1 & $1.025 \times 10^{-09}$ & $2.136 \times 10^{-09}$ & $1.712 \times 10^{-09}$ & $1.03 \times 10^{-09}$ & $9.97 \times 10^{-08}$ & $1.00 \times 10^{-09}$ & 136 & 2 \\
\hline 2 & $4.022 \times 10^{-11}$ & $1.045 \times 10^{-08}$ & $1.826 \times 10^{-09}$ & $4.02 \times 10^{-11}$ & $9.98 \times 10^{-08}$ & $1.00 \times 10^{-09}$ & 266 & 2 \\
\hline 3 & $2.362 \times 10^{-10}$ & $2.479 \times 10^{-09}$ & $3.789 \times 10^{-09}$ & $2.36 \times 10^{-10}$ & $9.97 \times 10^{-08}$ & $1.00 \times 10^{-08}$ & 490 & 3 \\
\hline 4 & $5.171 \times 10^{-11}$ & $6.570 \times 10^{-10}$ & $8.829 \times 10^{-11}$ & $5.17 \times 10^{-11}$ & $1.63 \times 10^{-07}$ & $1.00 \times 10^{-08}$ & 1000 & 5 \\
\hline 5 & $6.588 \times 10^{-11}$ & $9.399 \times 10^{-11}$ & $1.423 \times 10^{-10}$ & $6.59 \times 10^{-11}$ & $1.46 \times 10^{-07}$ & $1.00 \times 10^{-08}$ & 1000 & 5 \\
\hline
\end{tabular}




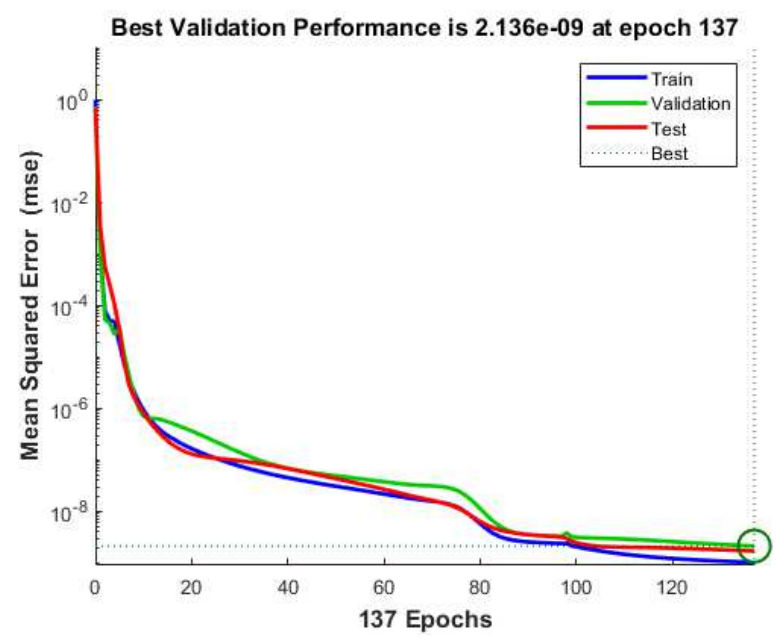

(a) Example 1: MSE performance

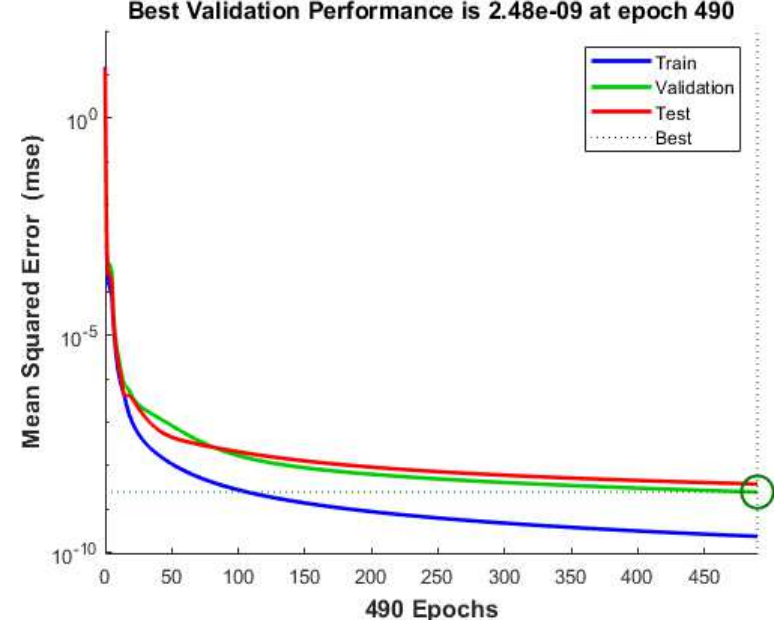

(c) Example 3: MSE performance

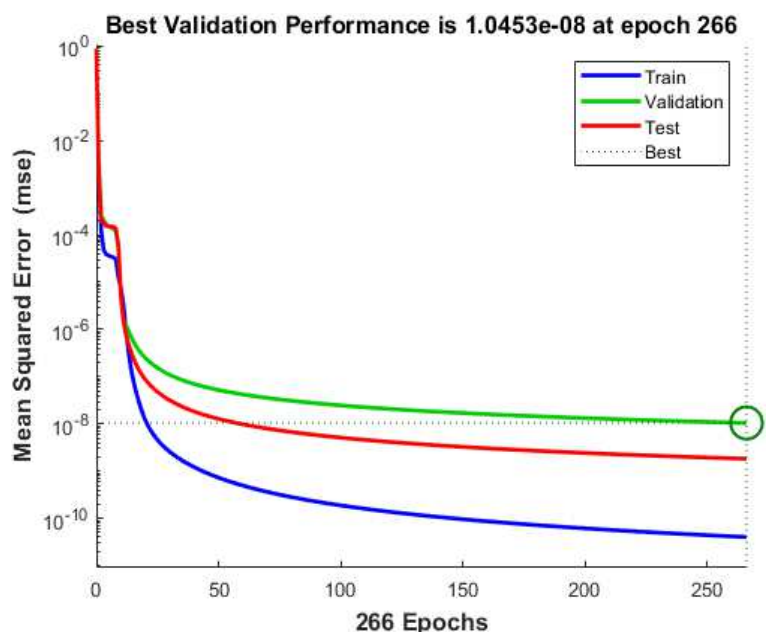

(b) Example 2: MSE performance

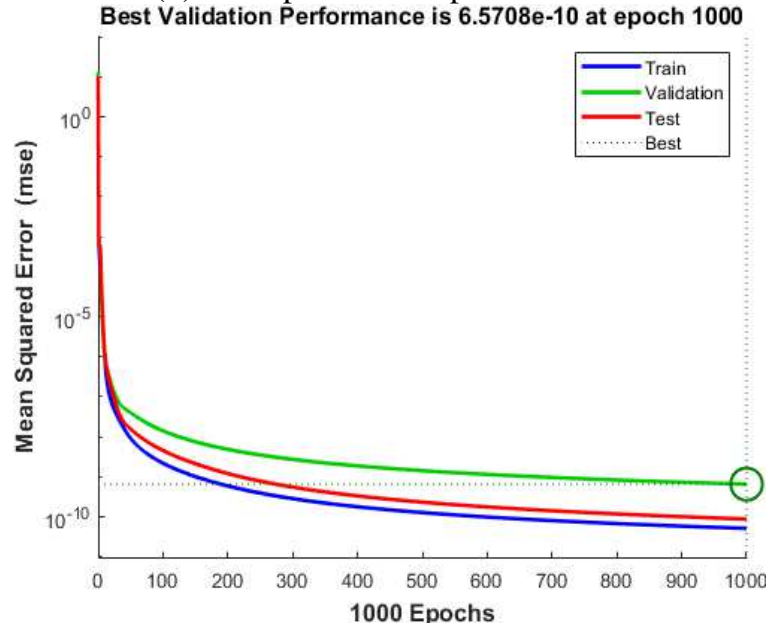

(d) Example 4: MSE performance

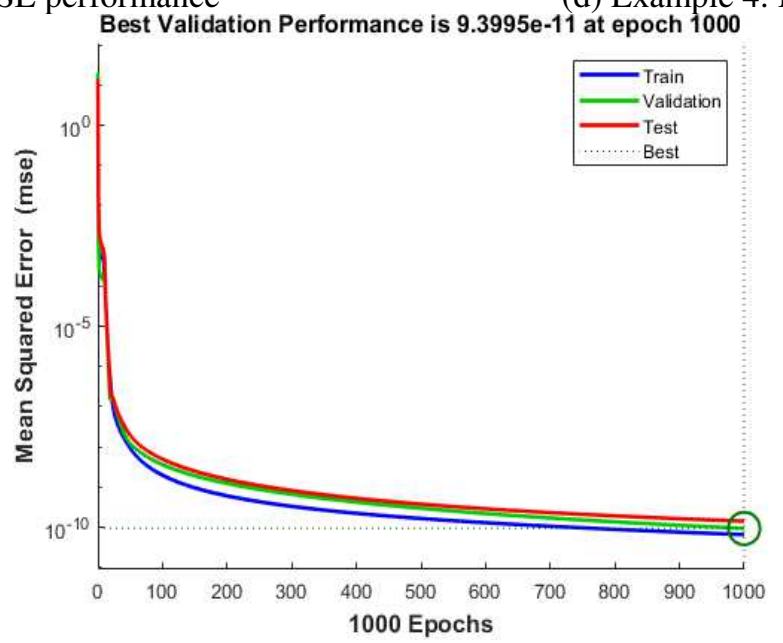

(e) Example 5: MSE performance

Fig 4: Curves based on the MSE Performances using the ANNs-LMB to solve the nonlinear novel SSO-PDS 

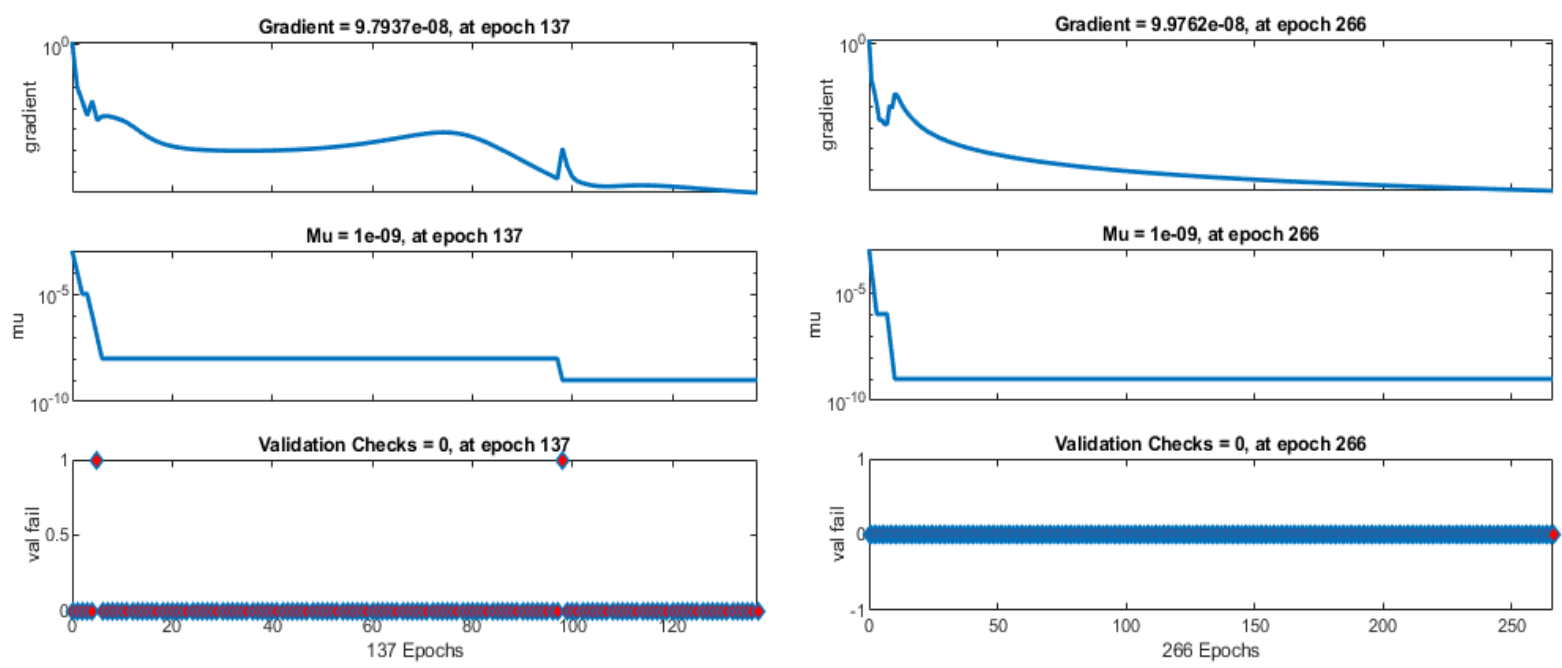

(a) Example 1: State transition

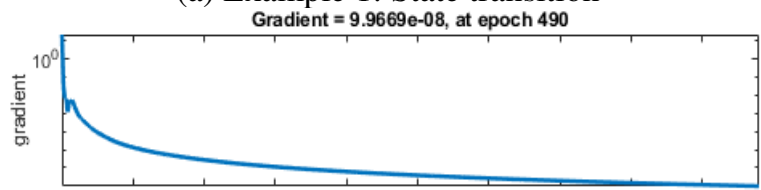

(b) Example 2: State transition
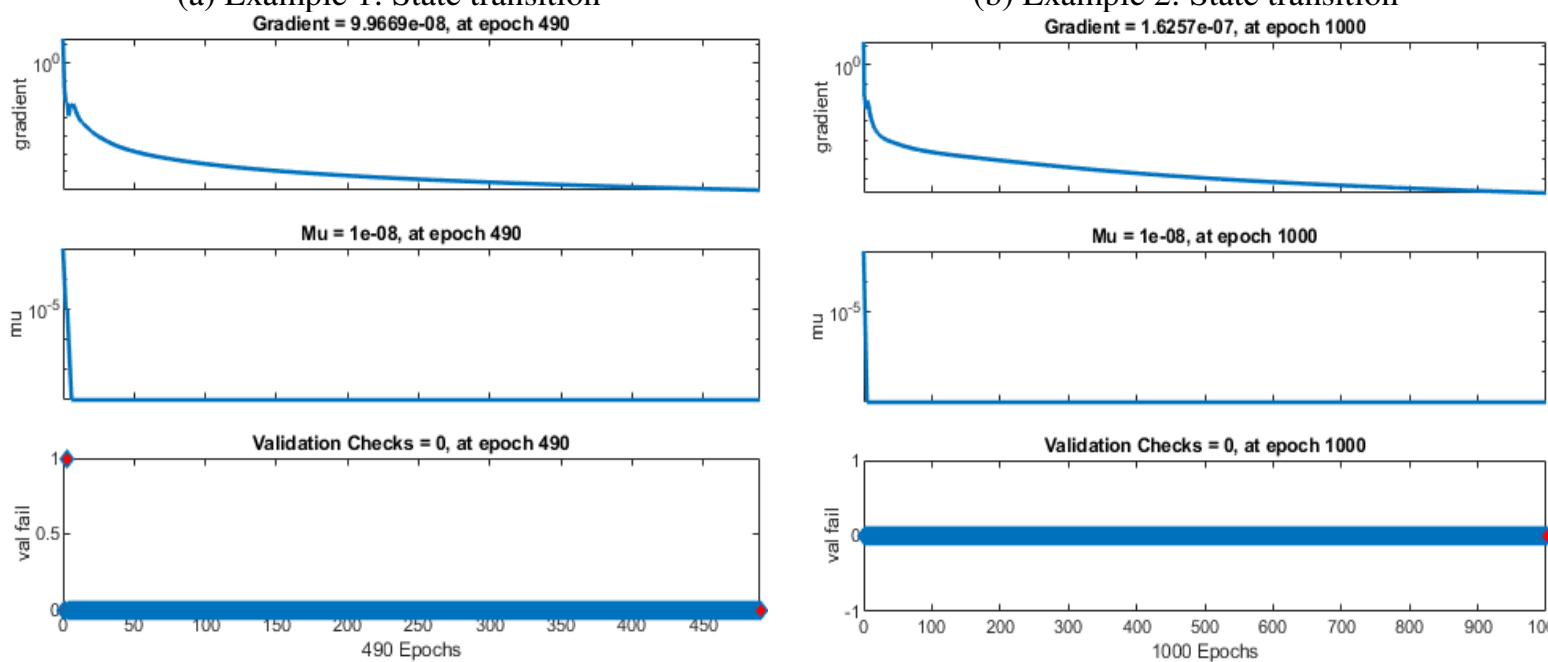

(c) Example 1: State transition

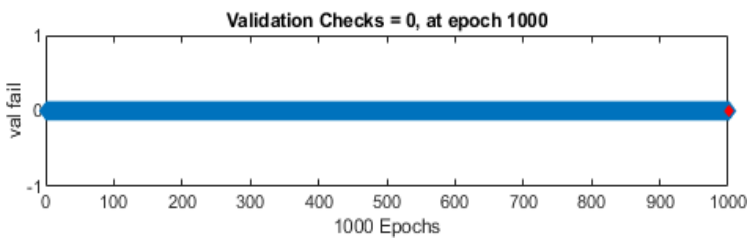

(d) Example 4: State transition
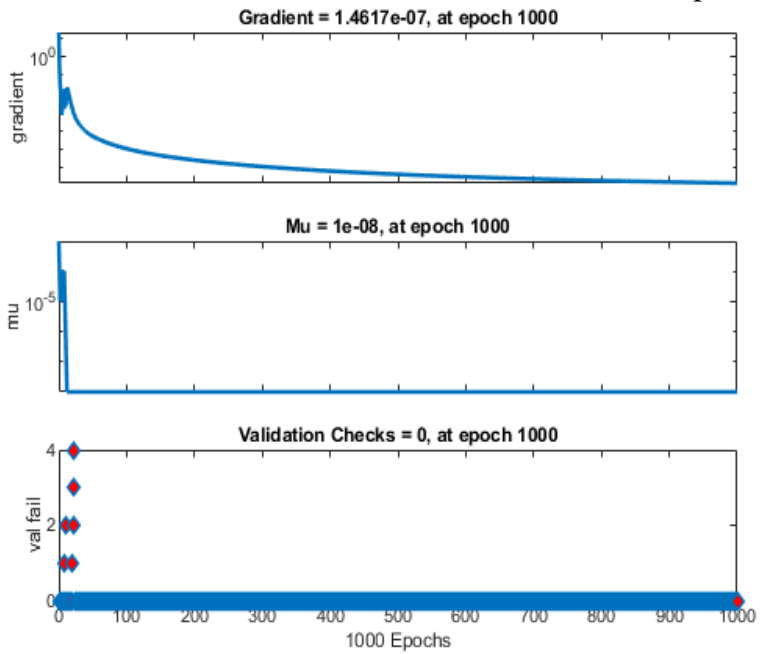

(e) Example 5: State transition

Fig 5: State transition curves using the ANNs-LMB to solve the nonlinear novel SSO-PDS 


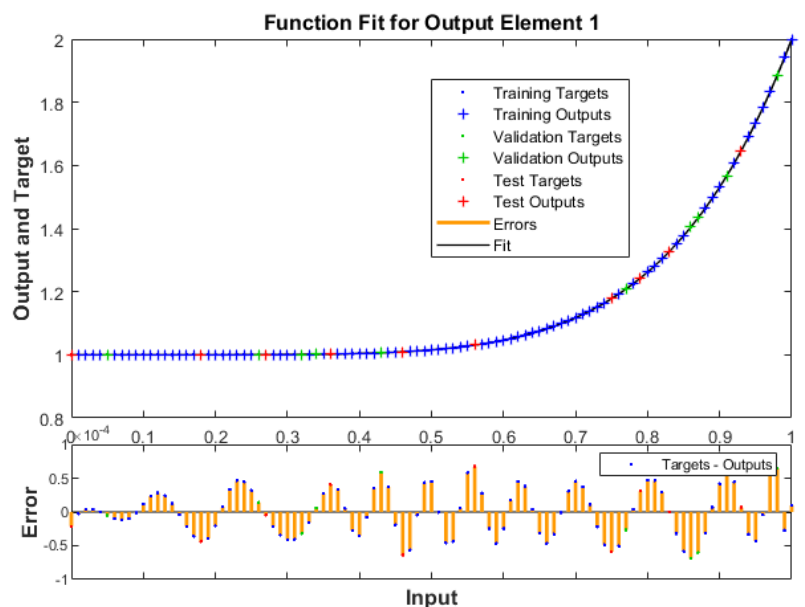

(a) Example 1: Result ccomparisons

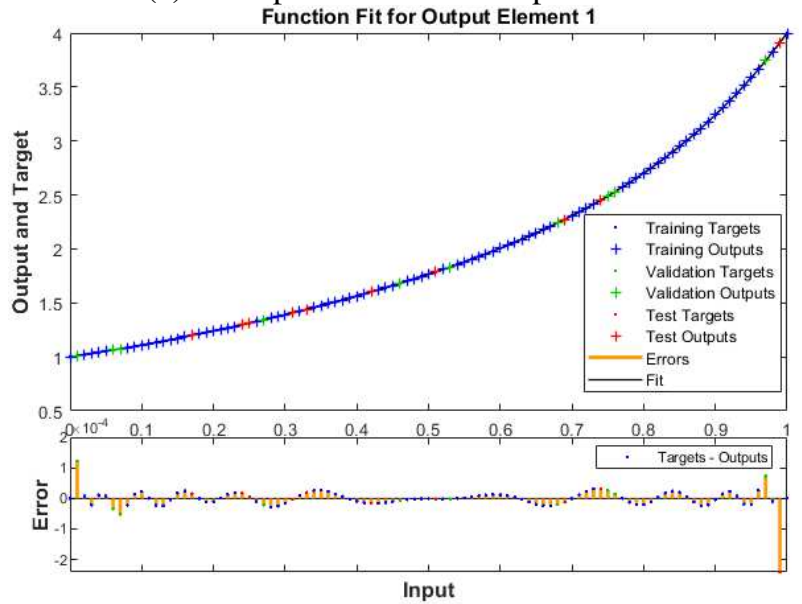

(c) Example 3: Result ccomparisons

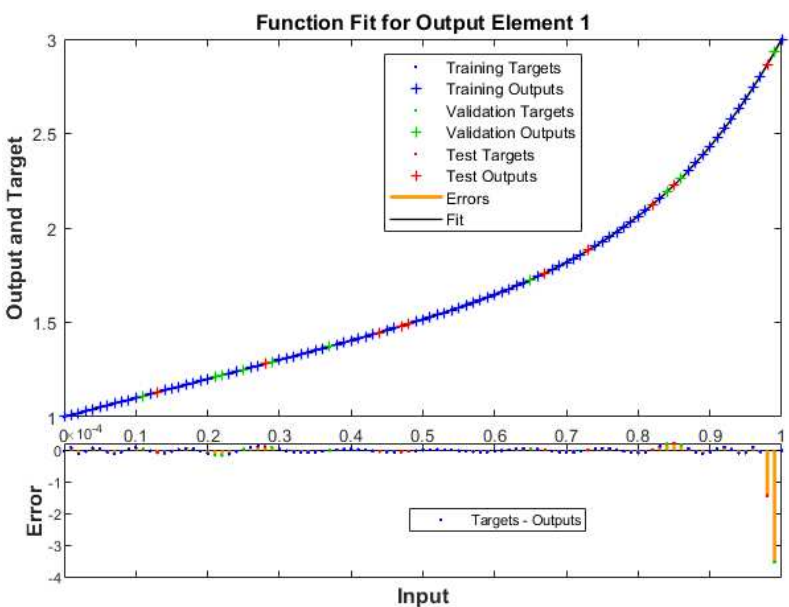

(b) Example 2: Result ccomparisons

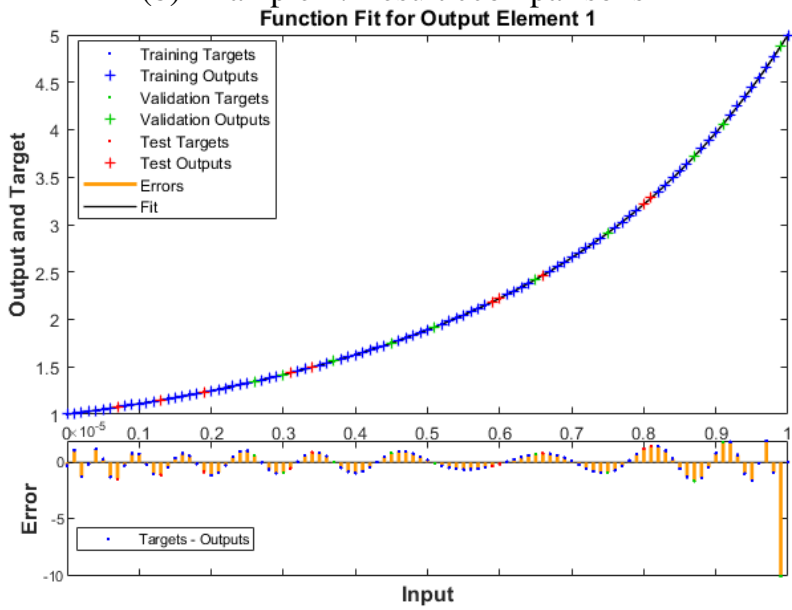

(d) Example 4: Result ccomparisons

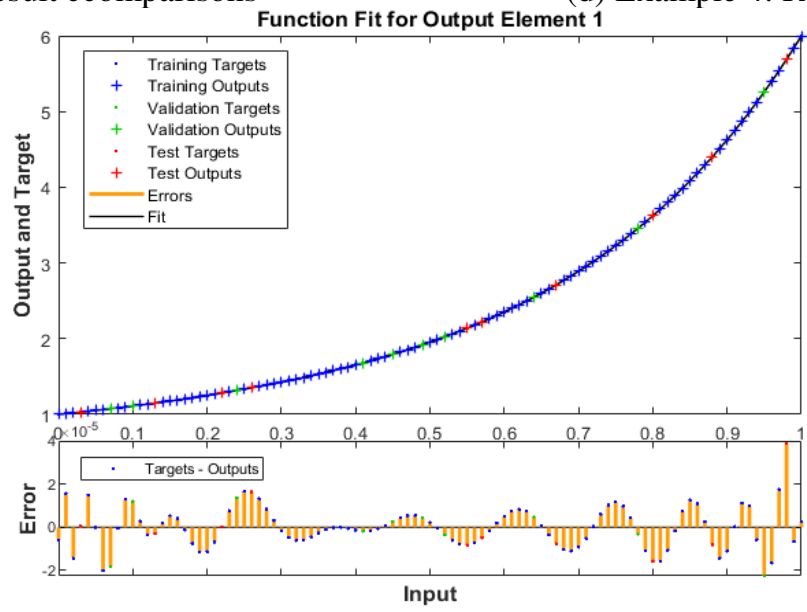

(e) Example 5: Result ccomparisons

Fig 6: Comparison of the results for each example of the nonlinear novel SSO-PDS 


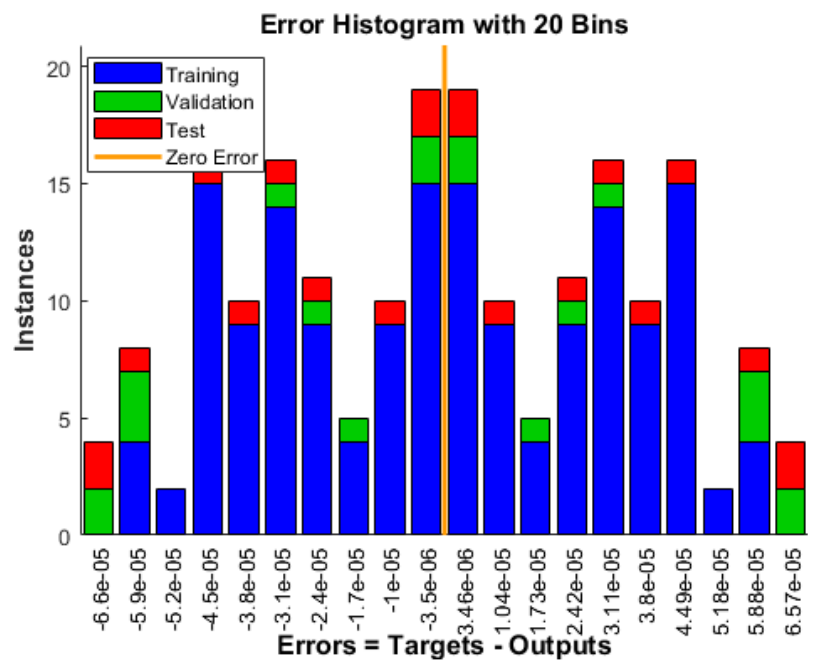

(a) Example 1: EHs

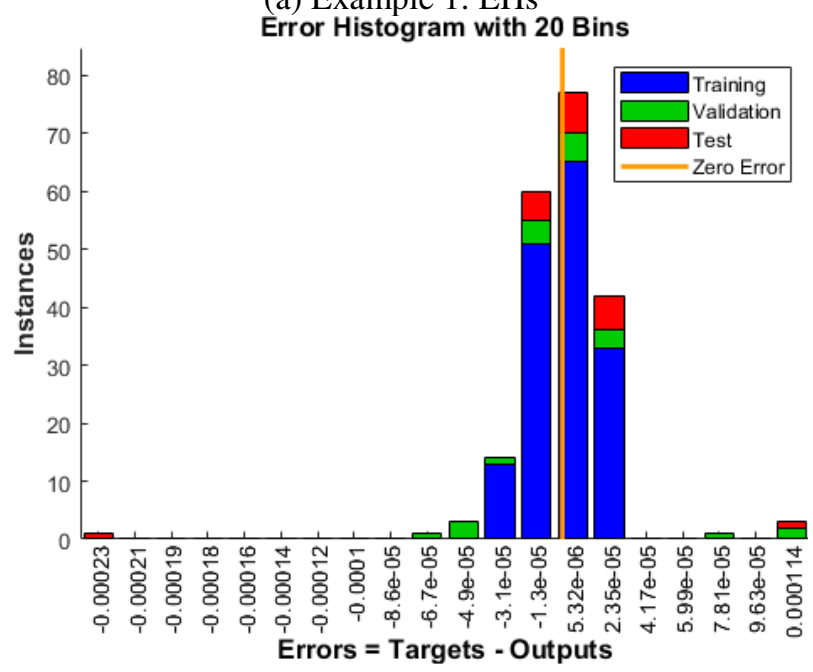

(c) Example 3: EHs

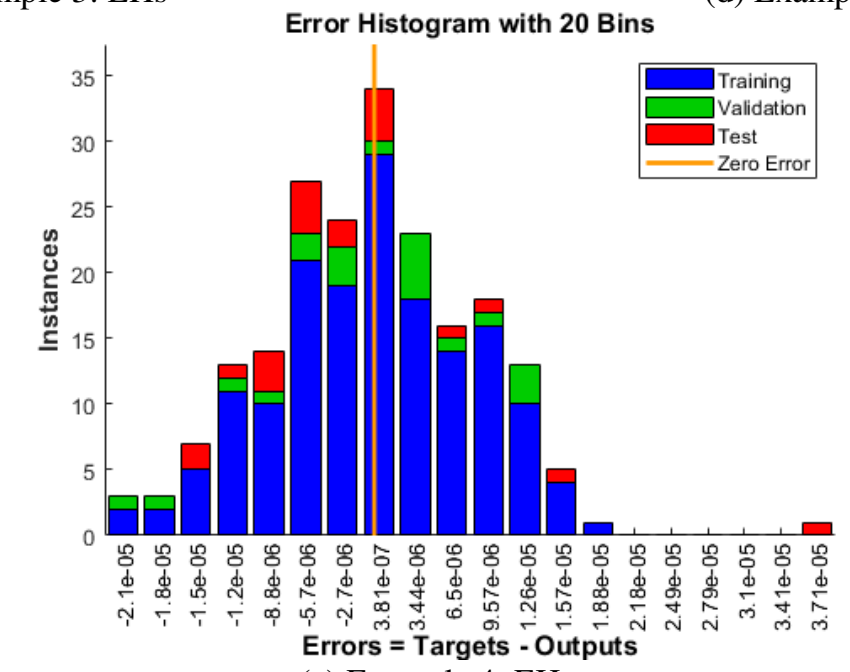

(e) Example 4: EHs

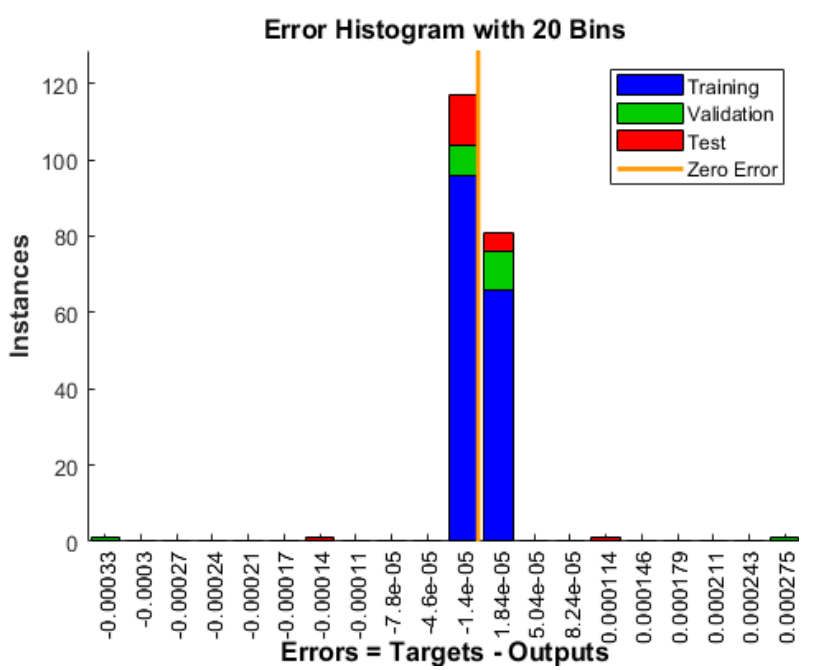

(b) Example 2: EHs

Error Histogram with 20 Bins

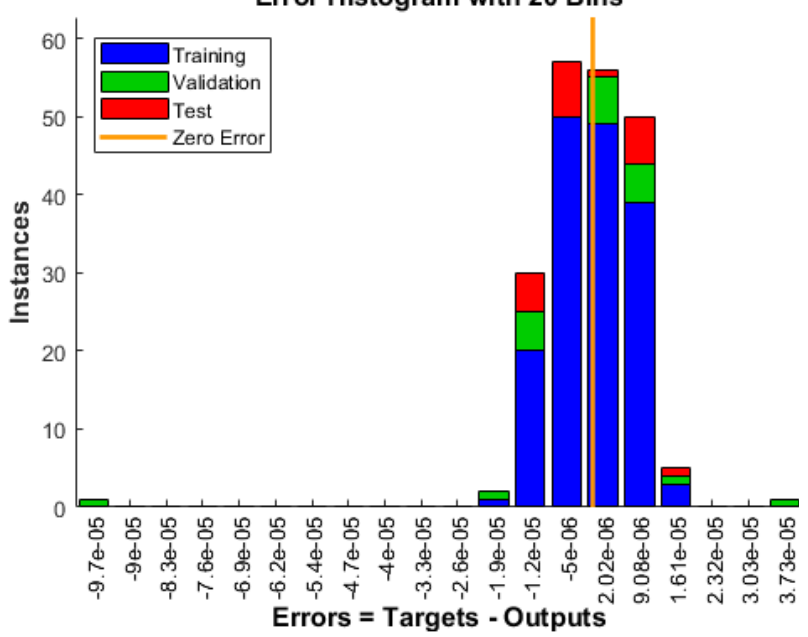

(d) Example 4: EHs

Fig 7: EHs for each example of the nonlinear novel SSO-PDS 

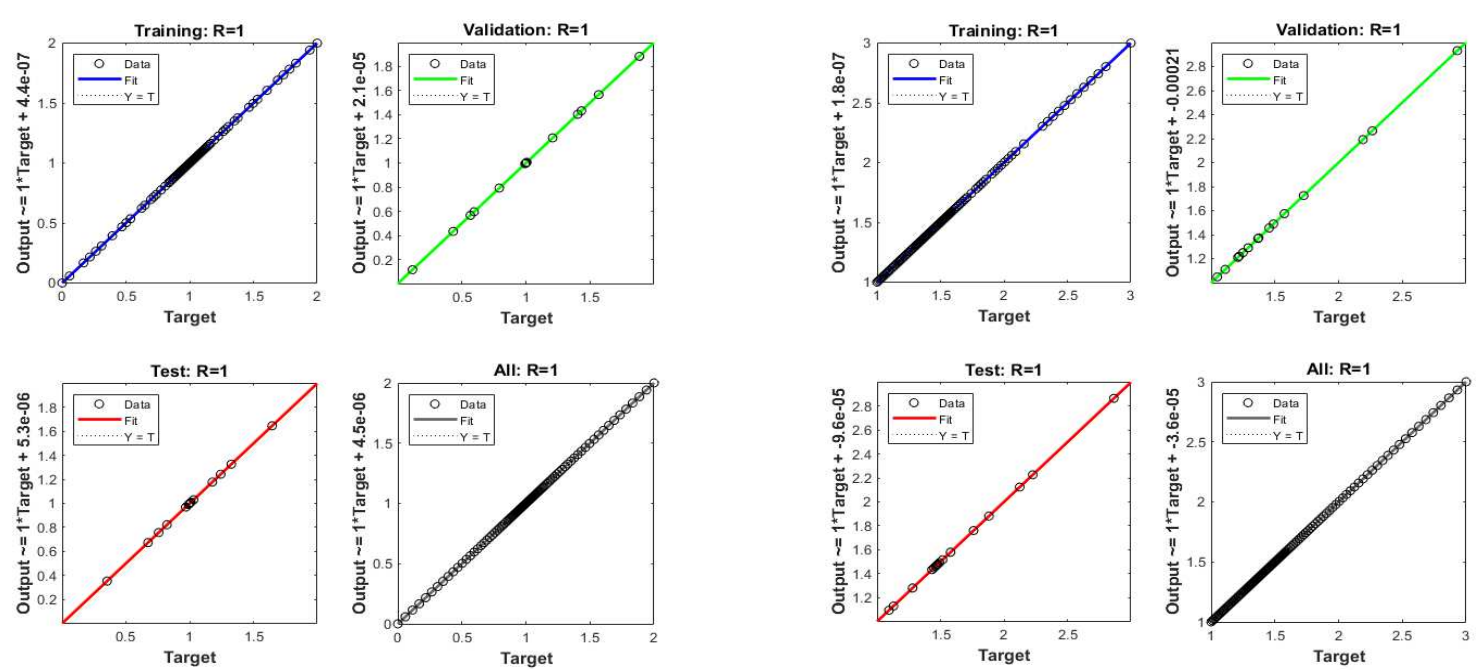

(a) Example 1: Regression plots
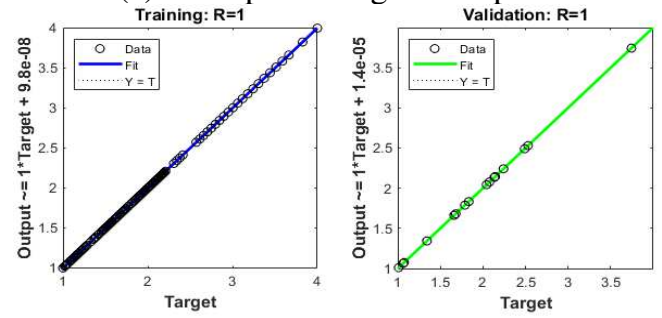

(b) Example 2: Regression plots
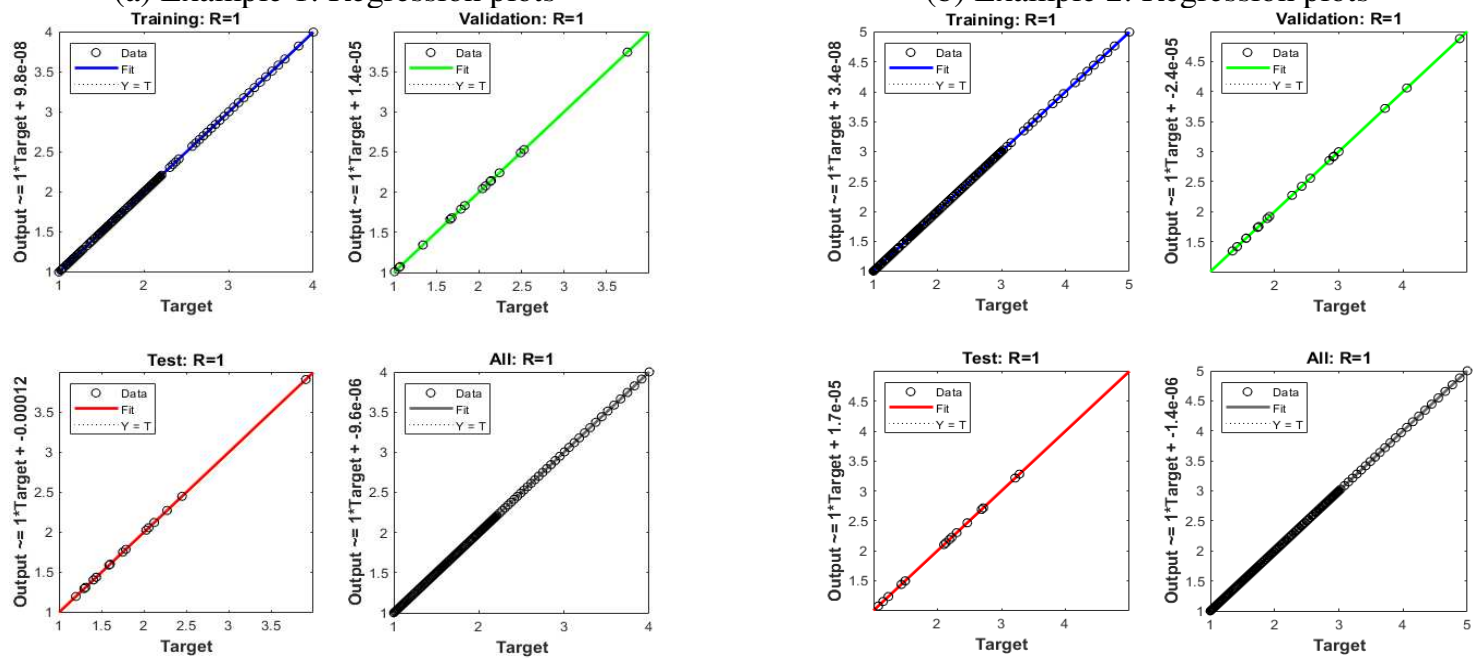

(c) Example 3: Regression plots
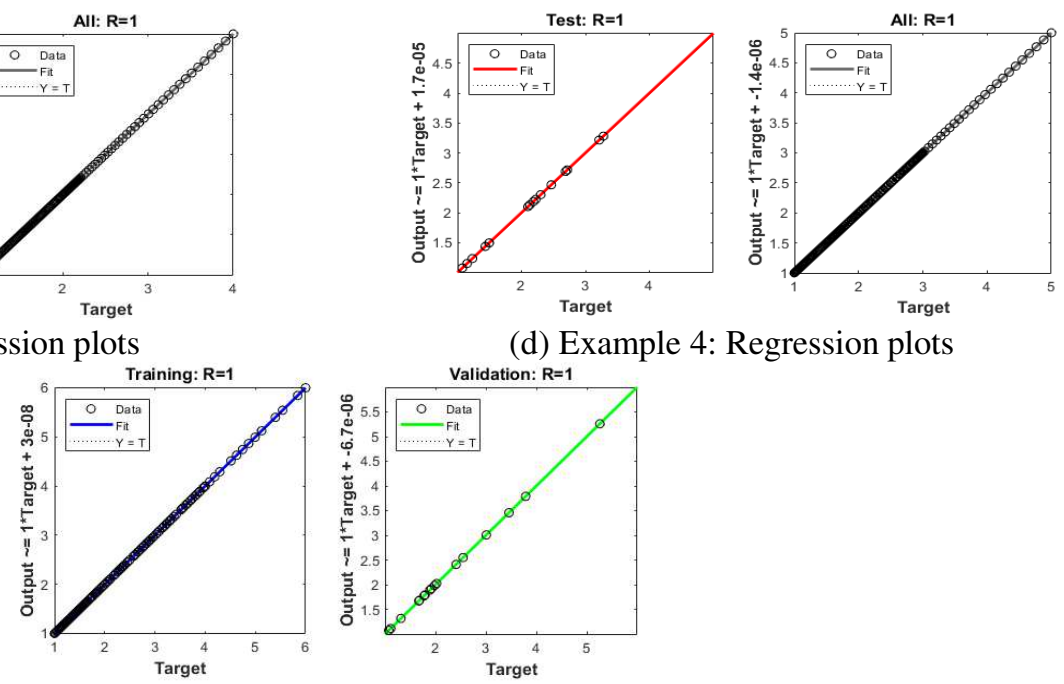

(d) Example 4: Regression plots
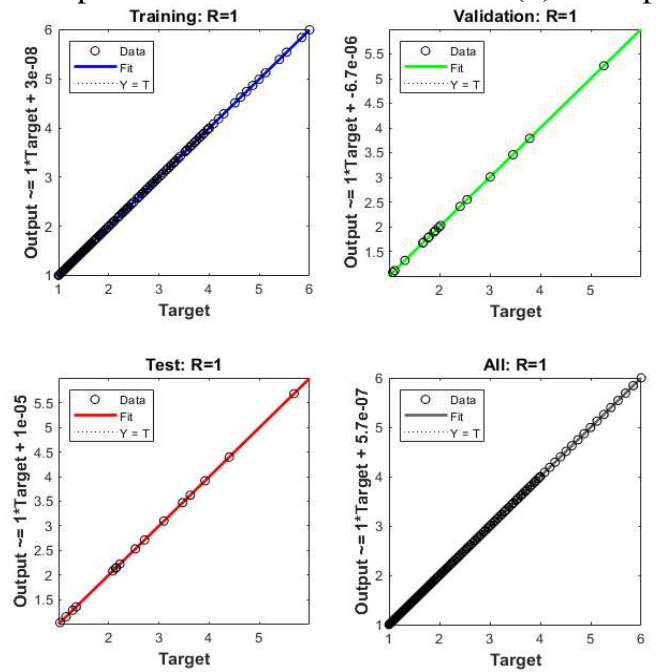

(e) Example 5: Regression plots

Fig 8: Regression plots for each example of the nonlinear novel SSO-PDS 
Figs 9 and 10 represent the comparison of the obtained outcomes and the reference solutions to solve each class of the nonlinear novel SSO-PDS. The results for both the categories $\omega_{1}(q)$ and $\omega_{2}(q)$ based on each class of the nonlinear novel SSO-PDS are represented in Fig 9. It is seen that the overlapping performances indicate the accuracy and exactness of the designed ANNs-LMB. The AE performances for each example of both the categories $\omega_{1}(q)$ and $\omega_{2}(q)$ based on the nonlinear novel SSO-PDS are provided in Fig 10. One can observe that the AE is found around $\left[10^{-04}, 10^{-07}\right]$ for each example of both the categories $\omega_{1}(q)$ and $\omega_{2}(q)$ based on the nonlinear novel SSO-PDS. These overlapping shows the correctness of the nonlinear novel SSO-PDS.

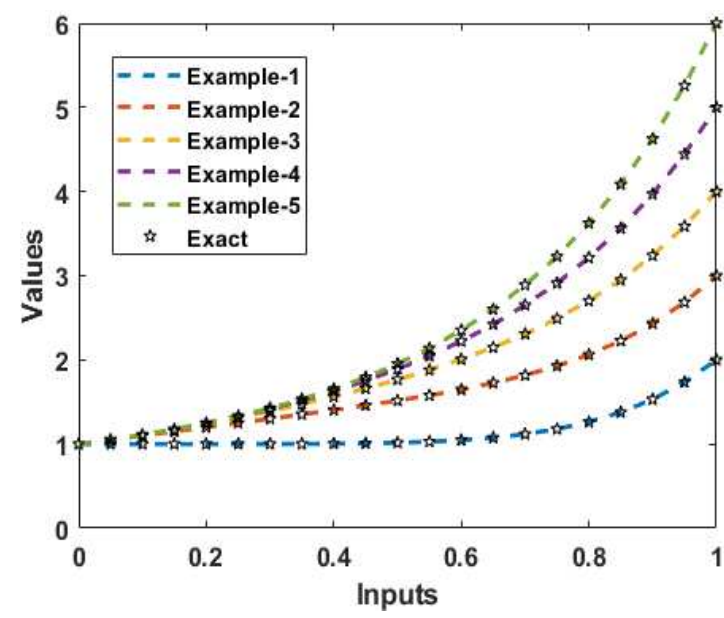

(a) Results of $\omega_{1}(q)$ for all examples

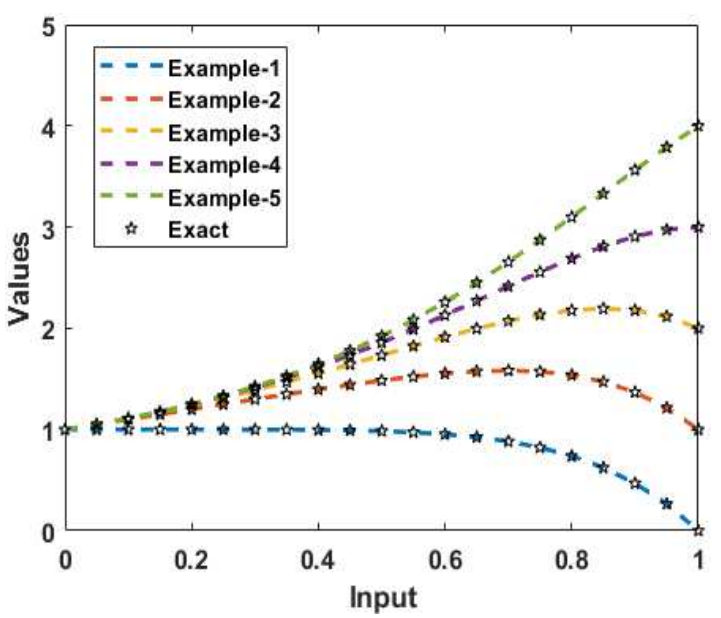

(b) Results of $\omega_{2}(q)$ for all examples

Fig 9: Results valuations for each example of the nonlinear novel SSO-PDS

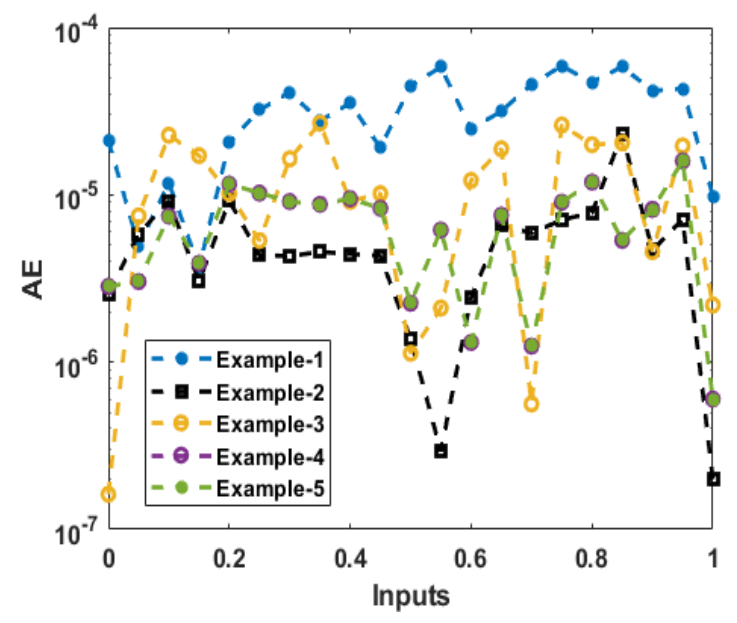

(c) $\mathrm{AE}$ of $\omega_{1}(q)$ for all examples

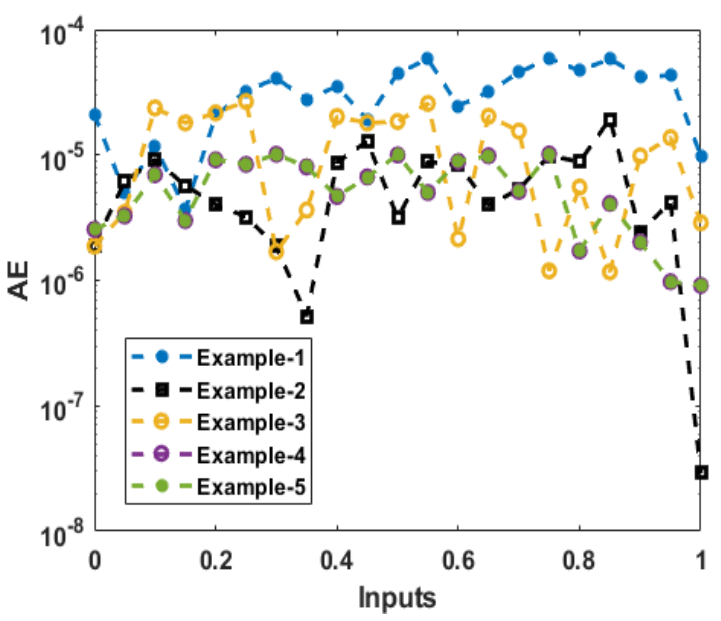

(d) $\mathrm{AE}$ of $\omega_{2}(q)$ for all examples

Fig 10: $\mathrm{AE}$ values for both of the parameters $\omega_{1}(q)$ and $\omega_{2}(q)$ for each example of the nonlinear novel SSO-PDS

\section{Conclusion}

The current study is related to design a novel nonlinear singular sixth order pantograph differential system along with its five categories. The design of the novel nonlinear SSO-PDS are presented using the concepts of the standard Emden-Fowler along with the pantograph differential system. 
The shape factor, singular point and pantograph terms are provided for each category of the novel nonlinear SSO-PDS. The novel model includes singular-points, multi-pantographs and nonlinear terms, which makes the model complex and stiffer in nature. In order to present the numerical solutions of the novel nonlinear SSO-PDS, an artificial neural network based LevenbergMarquardt backpropagation is proposed. The approximation statistics for training, validation and testing are $75 \%, 15 \%$ and $10 \%$ with the use of 15 hidden numbers of neurons based on the optimal performance. To authorize the precision of the novel nonlinear system, the overlapping plots have been performed and the values of AE are noticed in good measures. In order to authenticate the MSE performance, the convergence graphs of the verification, best curve, training and testing are observed based on the novel nonlinear SSO-PDS. The performances of correlation indorse the regression, whereas the gradient measures using the artificial neural network based LevenbergMarquardt backpropagation are calculated to solve novel nonlinear SSO-PDS. Furthermore, the correctness and accuracy are stated by using the regression dynamics, MSE and EHs.

In future, the design of artificial neural network based Levenberg-Marquardt backpropagation will be presented to solve the fluid problems, fractional order model and lonngren-wave equation and [54-60].

\section{Acknowledgments:}

We would thank the editing board and reviewers for their valuable response and fast reply that enhance the obtained results.

\section{Data Availability:}

The data used to support the findings of this study are available from the corresponding author upon request.

\section{Conflicts of Interest:}

The authors declare that they have no conflicts of interest.

\section{Authorship contribution statement:}

Zulqurnain Sabir: Formal analysis, Investigation, Software, Writing - original draft. Zulqurnain Sabir: Data curation, Validation, Visualization, Writing - review \& editing. Muhammad Asif Zahoor Raja: Investigation, Software. Muhammad Asif Zahoor Raja: Supervision. $\quad$ R. $\quad$ Sadat: Validation. $\quad$ R. $\quad$ Sadat: Resources. R. Sadat: Resources. Mohamed R. Ali: Project administration. Mohamed R. Ali: Conceptualization, Funding acquisition, Methodology, Software, Writing - review \& editing.

\section{Declaration of Competing Interest}

The authors declare that they have no known competing financial interests or personal relationships that could have appeared to influence the work reported in this paper. 


\section{References}

[1] Wazwaz, A.M., 2005. Adomian decomposition method for a reliable treatment of the EmdenFowler equation. Applied Mathematics and Computation, 161(2), pp.543-560.

[2] Sabir, Z., et al., 2021. Integrated intelligence of neuro-evolution with sequential quadratic programming for second-order Lane-Emden pantograph models. Mathematics and Computers in Simulation, 188, pp.87-101.

[3] Wong, J.S., 1975. On the generalized Emden-Fowler equation. Siam Review, 17(2), pp.339-360.

[4] Sabir, Z., et al., 2021. Neuro-evolution computing for nonlinear multi-singular system of third order Emden-Fowler equation. Mathematics and Computers in Simulation, 185, pp.799-812.

[5] Boubaker, $\mathrm{K}$ et al, 2012. Application of the BPES to Lane-Emden equations governing polytropic and isothermal gas spheres. New Astronomy, 17(6), pp. 565-569.

[6] Sabir, Z., et al., 2021. A neuro-swarming intelligent heuristic for second-order nonlinear LaneEmden multi-pantograph delay differential system. Complex \& Intelligent Systems, pp.1-14.

[7] Boubaker, $\mathrm{K}$ et al, 2012. Application of the BPES to Lane-Emden equations governing polytropic and isothermal gas spheres. New Astronomy, 17(6), pp. 565-569.

[8] Wazwaz, A.M., 2005. Adomian decomposition method for a reliable treatment of the EmdenFowler equation. Applied Mathematics and Computation, 161(2), pp.543-560.

[9] Wong, J.S., 1975. On the generalized Emden-Fowler equation. Siam Review, 17(2), pp.339-360.

[10] Taghavi, A et al, 2013. A solution to the Lane-Emden equation in the theory of stellar structure utilizing the Tau method. Mathematical Methods in the Applied Sciences, 36(10), pp.1240-1247.

[11] Sabir, Z., et al., 2020. Novel design of Morlet wavelet neural network for solving second order Lane-Emden equation. Mathematics and Computers in Simulation, 172, pp.1-14

[12] Adel, W et al, 2020. Solving a new design of nonlinear second-order Lane-Emden pantograph delay differential model via Bernoulli collocation method. The European Physical Journal Plus, 135(6), p.427. 4-100.

[13] Sabir, Z., et al., 2021. Solving a novel designed second order nonlinear Lane-Emden delay differential model using the heuristic techniques. Applied Soft Computing, 102, p.107105.

[14] Abbas, F., et al., 2019. Approximate solutions to lane-emden equation for stellar configuration. Applied Mathematics and Information Sciences, 13, pp.143-152.

[15] Singh, R., et al., 2019. Haar wavelet collocation approach for Lane-Emden equations arising in mathematical physics and astrophysics. The European Physical Journal Plus, 134(11), p.548.

[16] S. Chandrasekhar, An Introduction to the Study of Stellar Structure, Dover Publications, New York, 1967.

[17] Bhrawy, A. H et al., 2014, May. An efficient collocation method for a class of boundary value problems arising in mathematical physics and geometry. In Abstract and Applied Analysis (Vol. 2014). Hindawi Publishing Corporation.

[18] Khan, J. A et al., 2015. Nature-inspired computing approach for solving non-linear singular EmdenFowler problem arising in electromagnetic theory. Connection Science, 27(4), pp. 377-396.

[19] Luo, T et al., 2016. Nonlinear asymptotic stability of the Lane-Emden solutions for the viscous gaseous star problem with degenerate density dependent viscosities. Communications in Mathematical Physics, 347(3), pp. 657-702.

[20] Dehghan, M. et al., 2008. Solution of an integro-differential equation arising in oscillating magnetic fields using He's homotopy perturbation method. Progress in Electromagnetics Research, 78, pp. 361-376.

[21] Ramos, J. I., 2003. Linearization methods in classical and quantum mechanics. Computer Physics Communications, 153(2), pp. 199-208.

[22] Radulescu, $V$ et al., 2012. Combined effects in nonlinear problems arising in the study of anisotropic continuous media. Nonlinear Analysis: Theory, Methods and Applications, 75(3), pp. 1524-1530. 
[23] J. D \v\{z\}urina, S.R. Grace, I. Jadlovsk $\backslash\{a\}$, and T. Li, Oscillation criteria for second-order Emden-Fowler delay differential equations with a sublinear neutral term, Math. Nachr. 293 (2020), 1--13. https://doi.org/10.1002/mana.201800196.

[24] Taghavi, A et al, 2013. A solution to the Lane-Emden equation in the theory of stellar structure utilizing the Tau method. Mathematical Methods in the Applied Sciences, 36(10), pp.1240-1247.

[25] Sabir, Z., et al., 2020. A Neuro-Swarming Intelligence-Based Computing for Second Order Singular Periodic Non-linear Boundary Value Problems. Front. Phys, 8, p.224.

[26] Abdelkawy, M.A., et al, 2020. Numerical investigations of a new singular second-order nonlinear coupled functional Lane-Emden model. Open Physics, 18(1), pp.770-778.

[27] Sabir, Z., et al., 2021. Integrated intelligence of neuro-evolution with sequential quadratic programming for second-order Lane-Emden pantograph models. Mathematics and Computers in Simulation.

[28] Nisar, K, et al., 2021. Evolutionary Integrated Heuristicwith Gudermannian Neural Networks for Second Kind of Lane-Emden Nonlinear Singular Models. Appl. Sci. 2021, 11, 4725. https://doi.org/10.3390/app11114725

[29] Sabir, Z., et al., 2020. 'Intelligence Computing Approach for Solving Second Order System of Emden-Fowler Model. Journal of Intelligent \& Fuzzy Systems, vol. 38, no. 6, pp. 7391-7406.

[30] Parand, K. et al., 2004. Rational Legendre approximation for solving some physical problems on semi-infinite intervals. Physica Scripta, 69(5), p. 353.

[31] Sabir, Z., et al., 2021. Neuro-evolution computing for nonlinear multi-singular system of third order Emden-Fowler equation. Mathematics and Computers in Simulation, 185, pp.799-812.

[32] Guirao, J.L., et al., 2020. Design and numerical solutions of a novel third-order nonlinear EmdenFowler delay differential model. Mathematical Problems in Engineering, 2020.

[33] Sabir, $Z$ et al, 2020. Design of neuro-swarming-based heuristics to solve the third-order nonlinear multi-singular Emden-Fowler equation. The European Physical Journal Plus, 135(6), pp.1-17.

[34] Li, D.S. et al., 2000. Exact solution properties of a multi-pantograph delay differential equation. J Harbin Inst Technol, 32(3), pp.1-3.

[35] Kuang, Y. ed., 1993. Delay differential equations: with applications in population dynamics (Vol. 191). Academic press.

[36] Zhao, T., 1995. Global periodic-solutions for a differential delay system modeling a microbial population in the chemostat. Journal of mathematical analysis and applications, 193(1), pp.329352.

[37] Li, W., et al., 2014. Ultrafast all-optical graphene modulator. Nano letters, 14(2), pp.955-959.

[38] Niculescu, S.I., 2001. Delay effects on stability: a robust control approach (Vol. 269). Springer Science \& Business Media.

[39] Soleymani Karimi Vanani, et al. 2011. On the numerical solution of generalized pantograph equation. World Applied Sciences Journal, 13(12):2531-2535.

[40] Liu, M.Z. et al., 2004. Properties of analytic solution and numerical solution of multi-pantograph equation. Applied Mathematics and Computation, 155(3), pp.853-871.

[41] Sezer, M. et al., 2008. Approximate solution of multi-pantograph equation with variable coefficients. Journal of Computational and Applied Mathematics, 214(2), pp.406-416.

[42] Koroma, M.A., et al., 2013. Laplace decomposition approximation solution for a system of multipantograph equations. International Journal of Mathematical, Computational Science and Engineering, 7(7), pp.39-44.

[43] Keskin, Y., et al., 2007. Approximate solutions of generalized pantograph equations by the differential transform method. International Journal of Nonlinear Sciences and Numerical Simulation, 8(2), pp.159-164. 
[45] Abazari, N. et al., 2009, October. Solution of nonlinear second-order pantograph equations via differential transformation method. In Proceedings of World Academy of Science, Engineering and Technology (Vol. 58, pp. 1052-1056).

[44] Derfel, G. et al., 1997. The pantograph equation in the complex plane. Journal of Mathematical Analysis and Applications, 213(1), pp.117-132.

[46] Ramos, J.I., 2008. Series approach to the Lane-Emden equation and comparison with the homotopy perturbation method. Chaos, Solitons \& Fractals, 38(2), pp.400-408.

[47] Saeed, U., 2017. Haar Adomian method for the solution of fractional nonlinear Lane-Emden type equations arising in astrophysics. Taiwanese Journal of Mathematics, 21(5), pp.1175-1192.

[48] Hashemi, M.S., et al., 2017. Solving the Lane-Emden equation within a reproducing kernel method and group preserving scheme. Mathematics, 5(4), p.77.

[49] Sabir, Z., et al 2020. On a new model based on third-order nonlinear multi singular functional differential equations. Mathematical Problems in Engineering, 2020.

[50] Sabir, Z., et al., 2020. Heuristic computing technique for numerical solutions of nonlinear fourth order Emden-Fowler equation. Mathematics and Computers in Simulation, 178, pp.534-548.

[51] Sabir, Z., et al., FMNEICS: fractional Meyer neuro-evolution-based intelligent computing solver for doubly singular multi-fractional order Lane-Emden system. Comp. Appl. Math. 39, 303 (2020). https://doi.org/10.1007/s40314-020-01350-0.

[52] Sabir, Z., et al., 2020. Integrated intelligent computing paradigm for nonlinear multi-singular thirdorder Emden-Fowler equation. Neural Computing and Applications, pp.1-20.

[53] Sabir, Z., et al., 2020. Numerical investigations to design a novel model based on the fifth order system of Emden-Fowler equations. Theoretical and Applied Mechanics Letters, 10(5), pp.333342.

[54] Baskonus, H.M. et al., 2019. New complex hyperbolic structures to the lonngren-wave equation by using sine-gordon expansion method. Applied Mathematics and Nonlinear Sciences, 4(1), pp.141150.

[55] Brzeziński, D.W., 2018. Review of numerical methods for NumILPT with computational accuracy assessment for fractional calculus. Applied Mathematics and Nonlinear Sciences, 3(2), pp.487502.

[56] Duru, H., et al., 2020. New analytical solutions of conformable time fractional bad and good modified Boussinesq equations. Applied Mathematics and Nonlinear Sciences, 5(1), pp.447-454.

[57] Ilhan, E. and Kıymaz, I.O., 2020. A generalization of truncated M-fractional derivative and applications to fractional differential equations. Applied Mathematics and Nonlinear Sciences, 5(1), pp.171-188.

[58] Sajid, T., et al., 2020. Impact of activation energy and temperature-dependent heat source/sink on maxwell-sutterby fluid. Mathematical Problems in Engineering, 2020.

[59] Sajid, T., et al., 2021. Upshot of radiative rotating Prandtl fluid flow over a slippery surface embedded with variable species diffusivity and multiple convective boundary conditions. Heat Transfer, 50(3), pp.2874-2894.

[60] Sajid, T.et al., 2021. Impact of oxytactic microorganisms and variable species diffusivity on bloodgold Reiner-Philippoff nanofluid. Applied Nanoscience, 11(1), pp.321-333. 